\title{
Logical and experimental modeling of cytokine and eicosanoid signaling in psoriatic keratinocytes
}

\author{
Running title: Psoriatic keratinocytes modeling \\ Eirini Tsirvouli $^{1 *}$, Felicity Ashcroft ${ }^{1 *}$, Berit Johansen $^{1}$, Martin Kuiper ${ }^{1}$ \\ ${ }^{1}$ Department of Biology, Norwegian University of Science and Technology, Trondheim, Norway \\ *Equally contributing authors \\ Corresponding author: eirini.tsirvouli@ntnu.no
}

\begin{abstract}
Psoriasis is characterized by chronic inflammation, perpetuated by a Th17-dependent signaling loop between the immune system and keratinocytes that could involve phospholipase $\mathrm{A}_{2}\left(\mathrm{PLA}_{2}\right)$-dependent eicosanoid release. A prior knowledge network supported by experimental observations was used to encode the regulatory network of psoriatic keratinocytes in a computational model for studying the mode of action of a cytosolic (c) $\mathrm{PLA}_{2} \alpha$ inhibitor. A combination of evidence derived from the computational model and experimental data suggests that Th17 cytokines stimulate pro-inflammatory cytokine expression in psoriatic keratinocytes via activation of $\mathrm{cPLA}_{2} \alpha-\mathrm{PGE}_{2}-\mathrm{EP} 4$ signaling, which could be suppressed using the anti-psoriatic calcipotriol. $\mathrm{cPLA}_{2} \alpha$ inhibition and calcipotriol showed overlapping and distinct modes of action. Model analyses revealed the immunomodulatory role of Th1 cytokines, the modulation of the physiological states of keratinocytes by Th17 cytokines, and how Th1 and Th17 cells together promote the development of psoriasis. Model simulations additionally suggest novel drug targets, including EP4 and PRKACA, for treatment that may restore a normal phenotype. Our work illustrates how the study of complex diseases can benefit from an integrated systems approach.
\end{abstract}

Keywords: cPLA2 / eicosanoids / logical modeling / psoriasis

\section{Introduction}

Psoriasis is a chronic inflammatory disease that affects $2-3 \%$ of the world's population. Psoriasis vulgaris, commonly referred to as plaque-type psoriasis, is the most prominent type of the disease accounting for $90 \%$ of total cases (Boehncke \& Schön, 2015). The characteristic histological changes of the disease are the hyperproliferation and aberrant differentiation of keratinocytes $(\mathrm{KCs})$, together with the inflammatory state and infiltration of immune cells in the dermis and epidermis (Meephansan et al, 2017). While the infiltration of immune cell subpopulations, namely dendritic cells (DCs), T-lymphocytes, neutrophils and mast cells, were for a long time considered the main pathogenic factor of psoriasis, the key role of KCs in the initiation and the maintenance of the chronic nature of the disease is indisputable (Lowes et al, 2013). Therefore, the disease appears to be a result of a rather complex interaction between different cell types (Georgescu et al, 2019; Benhadou et al, 2019; Albanesi et al, 2018; Deng et al, 2016) and should be 
studied as a multiscale dysregulation. The understanding of the complex dynamics of psoriasis, and the identification of effective treatments may benefit from an integrative approach and the construction of systems-based models.

Psoriasis can be divided into two main stages: initiation and maintenance/chronic stage. In early events, $\mathrm{KCs}$ respond to different environmental and genetic stimuli by secreting immune mediators that result in the recruitment of DCs to the dermis and epidermis and their subsequent maturation. Activated DCs are then able to secrete cytokines that influence the differentiation of naive T-cells (Albanesi et al, 2018). In psoriasis, three T-helper (Th) cell subpopulations have well-documented roles in the progression and maintenance of the disease: Th1, Th17, and Th22 cells. Each Th type contributes to the cytokine milieu of psoriatic lesions. Th1 cells release IFN $\gamma$ and TNF $\alpha$, while Th17 produce IL-17 and IL-22, partially overlapping with the role of Th22 cells that produce only IL-22 (Benham et al, 2013, 22). The activation of these three classes of immune cells, in turn, triggers KCs to further produce antimicrobial peptides (AMPs), proinflammatory cytokines, and chemoattractants, which all together recruit neutrophils and maintain the same Th-cell subpopulation in a positive feedback loop that sustains chronic inflammation (Georgescu et al, 2019; Albanesi et al, 2018; Deng et al, 2016).

The chronic, life-long nature of psoriasis requires long-term treatment plans that are able to cope with the relapsing episodes of the disease (Gisondi et al, 2017). The latest advances in our understanding of the disease have led to the development of biologics targeting specific immune components (cells or proteins) (Kamata \& Tada, 2020). Other treatment options include vitamin D analogues such as calcipotriol, which affects KCs and immune cells (Kim, 2010). Current treatment options appear to have relatively high efficacy and tolerability, however, immediate or long-lasting efficacy can vary between patients (Kamata \& Tada, 2020; Kim, 2010), and certain biologics are accompanied by side effects and tolerability issues such as recurrent infections (Kamata \& Tada, 2020). Combination treatment with two or more agents has been proposed as a way to overcome these challenges and improve efficacy in non-responding patients while ameliorating and reducing the potential side effects of long-term treatments (Cather \& Crowley, 2014).

The dysregulated hydrolysis of phospholipids catalyzed by the phospholipase A2 (PLA2) enzyme family is involved in multiple diseases, including cancer, rheumatoid arthritis, and psoriasis (Magrioti \& Kokotos, 2013; Leistad et al, 2011; Linkous \& Yazlovitskaya, 2010). Cytosolic PLA2a (cPLA $2 \alpha$ ) hydrolyzes phosphatidylcholine (PC) to yield lysophosphatidylcholine (LPC) and arachidonic acid (AA), two bioactive lipids, the latter of which can be further metabolized by cyclooxygenase (COX) and lipoxygenase (LOX) enzymes to produce prostaglandins (PGs), leukotrienes (LTs) and hydroxy fatty acids e.g. 12s-HETE. These AA metabolites, or eicosanoids, are involved in various diseases, often producing inflammatory responses, but can also be involved in the activation of survival-promoting kinases (Linkous \& Yazlovitskaya, 2010; Hirabayashi et al, 2004; Murakami et al, 2017) and cell cycle progression (Naini et al, 2015, 2016).

The signaling through $\mathrm{cPLA}_{2} \mathrm{a}$ and its downstream lipid mediators has a prominent role in different stages of psoriasis, with epidermal KCs being an important source of eicosanoids, including prostanoids and leukotrienes (Nicolaou, 2013), which similarly participate in the infiltration and amplification of certain immune cell types (Ueharaguchi 2018, Camp et al 1984; Sheibanie 2004; Lee, 2019) and act upon the KCs themselves via multiple prostanoid and leukotriene receptors (Konger 1998, Konger 2005; Kanda 2007, Kim 2010.) While Th1 cytokines are established activators of phospholipases and cause eicosanoid release from KCs (Sjursen et al, 2000; Thommesen et al, 1998) the effects of Th17 cytokines on 
eicosanoid production and signaling is unclear. Furthermore, $\mathrm{PGE}_{2}$ has been reported to regulate Th17 and Th1 differentiation and response (Boniface et al, 2009; Napolitani et al, 2009; Yao et al, 2009), as well as affecting DCs (Chizzolini \& Brembilla, 2009). PGE 2 acts through four receptors, EP1-EP4, all expressed in KCs (Rundhaug et al, 2011). The main receptor involved in psoriasis is EP4 (Tsuge et al), which activates both the AC/cAMP pathway and the PI3K/AKT pathway (Rundhaug et al, 2011). While the roles of EP1 and EP3 in psoriasis have not been well studied, their associated phenotypes do not appear to be characteristic for the disease as EP1 was found to promote differentiation in non-melanoma KCs (Konger et al, 2009) and EP3 was proposed as a growth inhibitor for KCs (Konger et al, 2005).

The targeting of $\mathrm{CPLA}_{2} \mathrm{a}$ with chemical inhibitors has been suggested as a promising treatment against hyperproliferative and inflammatory diseases (Yarla et al, 2016), also supported by the promising results in clinical trials against mild to moderate psoriasis (Omland et al, 2017). However, the limited understanding of the exact involvement of $\mathrm{CPLA}_{2} \mathrm{a}$ in psoriasis, as well as the general gaps of knowledge of its involvement in the development of the disease, require a unified framework that collects prior knowledge and integrates new observations to produce a modeling tool that can be further used to not only make sense of observations but also rationalize experimental decisions, test hypotheses and highlight gaps of knowledge. For this reason, in silico approaches to predict and interpret the effects of various stimuli and perturbations in cellular systems have become increasingly relevant. Among different possible approaches, logical models that represent the signaling events and causal interactions between cellular components prove to be able to capture the behavior of cells upon various experiments, predict the effect of drugs, and identify potentially synergistic combinations. (Tsirvouli et al, 2020; Pirkl et al, 2016; Flobak et al, 2015).

In this study, we aimed to develop such a logical model to represent the complex signaling events occurring in psoriatic keratinocytes to (1) investigate the mode of action of a cPLA ${ }_{2} \mathrm{a}$ inhibitor (2) predict therapeutic benefits of drug combinations, and (3) investigate the contributions of Th17 versus Th1 cytokines to disease phenotypes.

\section{Methods}

\section{Section I: In vitro methods}

\subsubsection{Materials}

The reagents used for culturing cells, and other chemicals, including calcipotriol hydrate were purchased from Sigma-Aldrich (St. Louis, MPS, US) unless stated otherwise. The fluoroketone AVX001 was synthesized and characterized according to Holmeide and Skattebol and provided by Dr. Inger Reidun Aukrust and Dr. Marcel Sandberg (Synthetica AS, Norway). AVX001 was stored at $-80{ }^{\circ} \mathrm{C}$ as a $20 \mathrm{mM}$ stock solution in DMSO under argon gas to minimize oxidation. Interleukins 17A (\#8928SC) and 22 (\#8931SC) were purchased from Cell Signalling Technologies. Recombinant human epidermal growth factor (EGF) was from R\&D Systems (Abingdon, UK).

\subsubsection{Maintenance of HaCaT Keratinocytes}

The spontaneously immortalized skin KC cell line HaCaT (Boukamp et al, 1988) was kindly provided by Prof. N. Fusenig (Heidelberg, Deutsches Krebsforschungszentrum, Germany). The cells were maintained in Dulbecco's modified Eagle Medium (DMEM) supplemented with 5\% (v/v) FBS, $0.3 \mathrm{mg} / \mathrm{mL}$ glutamine, and $0.1 \mathrm{mg} / \mathrm{mL}$ gentamicin (DMEM-5) at $37{ }^{\circ} \mathrm{C}$ with $5 \% \mathrm{CO} 2$ in a humidified atmosphere at sub-confluency. HaCaT are commonly used cell system to study proliferative and inflammatory responses 
in psoriasis research (Farkas et al, 2003; Feuerherm et al, 2013; Lehmann, 1997; Pozzi et al, 2004; Se et al, 2010; Ziv et al, 2008); the cells differentiate on reaching confluency and form stratified epithelia when grown under appropriate conditions (Hinitt et al, 2011; Schoop et al, 1999)

\subsubsection{D Culture of HaCaT Keratinocytes}

3D culture of HaCat KCs was carried out using the 24-well carrier plates system from Thermo Fisher Scientific (\#141002) according to the protocol described (Ashcroft et al, 2020). Briefly, the culture inserts $(0.4 \mu \mathrm{m}$ pore size) were coated using the Coating Matrix kit (Thermofisher Scientific \#R-011-K) according to the manufacturer's protocol. HaCaT were seeded at a density of $0.3 \times 10^{5}$ cells per insert in $0.5 \mathrm{~mL}$ DMEM-5. and incubated for $24 \mathrm{~h}$ before being lifted to the air-liquid interface. The media in the lower chamber was replaced with DMEM-5 (without antibiotics) $+1 \mathrm{ng} / \mathrm{mL}$ EGF, and $5 \mu \mathrm{g} / \mathrm{mL}$ L-ascorbic acid in the absence or presence of Th17 psoriatic cytokines (IL-17, $10 \mathrm{ng} / \mathrm{ml}$ and IL-22, 10 $\mathrm{ng} / \mathrm{ml})$ and either AVX001 $(5 \mu \mathrm{M})$, calcipotriol $(10 \mathrm{nM})$ or a combination of AVX001 $(5 \mu \mathrm{M})$ and calcipotriol $(10 \mathrm{nM})$. The media in the lower chambers was changed every 3rd day for 12 days.

\subsubsection{RNA Extraction and Real-Time QuantitativePCR}

Total RNA was extracted from HaCaT cultures using the RNeasy kit (QIAGEN) according to the manufacturer's protocol. The amount and purity of the RNA samples were quantified using a Nanodrop One/OneC Microvolume UV-VIS Spectrophotometer (ND-ONE-W) from ThermoFisher Scientific. RNA samples with absorbance (A) 260/280 between 2.0 and 2.2 were accepted. Reverse transcription was carried out using the QuantiTect Reverse Transcription Kit (Qiagen, Hilden, Germany) according to the manufacturer's protocol, using $1 \mu \mathrm{g}$ of total RNA per sample. Real-time PCR analysis was performed using the LightCycler 480 SYBR Green I Master MIX and LightCycler 96 instrument from Roche (Basel, Switzerland), according to the manufacturer's protocol with a final individual primer concentration of 0.5 $\mu \mathrm{M}$. Amplification was performed with an initial step of $95{ }^{\circ} \mathrm{C}(10 \mathrm{~min})$ followed by 45 cycles of denaturation at $95^{\circ} \mathrm{C}(10 \mathrm{~s})$, annealing at $55^{\circ} \mathrm{C}(10 \mathrm{~s})$ and elongation at $72{ }^{\circ} \mathrm{C}(10 \mathrm{~s})$. Melting analysis was performed with the following parameters: $95^{\circ} \mathrm{C}$ for $5 \mathrm{~s}, 65^{\circ} \mathrm{C}$ for $1 \mathrm{~min}, 97^{\circ} \mathrm{C}$ for $1 \mathrm{~min}$ and cooling at $50{ }^{\circ} \mathrm{C}$ for $10 \mathrm{~s}$. Three reference genes were selected for gene expression analysis: TBP, HPRT1, and GAPDH (Allen et al, 2008).) All primers were KiCqStart primers designed by and purchased from Sigma Aldrich. Ct values and PCR amplification efficiencies were calculated using LinRegPCR software (version 2017.1) (Ruijter et al, 2014; Tuomi et al, 2010) and imported into qBASE+ (version 3.2) (Hellemans et al, 2007) for relative expression analysis.

\subsubsection{Immunohistochemistry for analysis of proliferation and differentiation markers}

$\mathrm{HaCaT}$ cultures were processed in parallel to quantify the expression of ki67 and cytokeratin 10 as determinants of the proportion of proliferating cells and cells undergoing differentiation. These cultures were fixed by immersion in 4\% paraformaldehyde (PFA) overnight; the membranes were removed from the inserts and prepared in Tissue Clear (Sakura, Osaka, Japan) for paraffin wax embedding using the Excelsior AS Tissue processor (ThermoFisher Scientific). Paraffin-embedded sections $(4 \mu \mathrm{m})$ were cut onto SuperFrost Plus slides (ThermoFisher scientific), dried overnight at $37{ }^{\circ} \mathrm{C}$, and then baked for 60 min at $60{ }^{\circ} \mathrm{C}$. The sections were dewaxed in Tissue Clear and rehydrated through graded alcohols to water in an automatic slide stainer (Tissue-Tek Prisma, Sakura). Next, the sections were pretreated in Target Retrieval Solution, High pH (Dako, Glostrup, Denmark, K8004) in PT Link (Dako) for 20 min at $97{ }^{\circ} \mathrm{C}$ to facilitate antigen retrieval. The staining was performed according to the manufacturer's procedure with EnVision G|2 Doublestain System Rabbit/Mouse (DAB+/Permanent Red) kit (Dako/Agilent K5361) on the Dako Autostainer. Endogenous peroxidase and alkaline phosphatase activity were quenched with Dual 
Endogenous Enzyme Block (Dako). Sections were then rinsed in wash buffer and incubated with primary antibody against Ki67 (MIB1 (Dako M7240) diluted 1:300) for $40 \mathrm{~min}$. The slides were rinsed before incubating in horseradish peroxidase (HRP) - polymer and 3,3'-Diaminobenzidine (DAB) to develop the stain. After a double stain block, the sections were incubated in antibody against cytokeratin 10 (Invitrogen \#MA5-13705 diluted 1:100) for $60 \mathrm{~min}$. After incubation in the mouse/rabbit linker, the sections were incubated in AP- polymer and the corresponding red substrate buffer with washing between each step. Tris-buffered saline (TBS; Dako K8007) was used throughout the washing steps. The slides were lightly counterstained with hematoxylin, completely dried, and coverslipped. Appropriate negative controls were performed; both mouse monoclonal isotype control (Biolegend, San Diego, CA) and omitting the primary antibody (negative method control). Images were taken at 40X magnification and $>20$ images were typically collected per sample for counting.

\subsubsection{Enzyme-Linked Immunoassay Detection of Eicosanoids}

Supernatants from the $\mathrm{HaCaT}$ cultures were analyzed by enzyme-linked immunosorbent assay (ELISA) for $\mathrm{PGE}_{2}$ (Cayman \#514435), $\mathrm{LTB}_{4}$ (Cayman \#10009292), TxB (Cayman \#501020), and 12s-HETE (Enzo Life Sciences \#ADI-900-050) according to the manufacturer's protocols. Cell supernatants were assayed at dilutions of 1:100 for $\mathrm{PGE}_{2}$, except supernatants from non-LPS-treated PBMC that were assayed undiluted in all assays. Supernatants were hybridized overnight, and the enzymatic conversion of the substrate was read at OD420 nm. Data were processed using a 4-parameter logistic fit model.

\subsubsection{Experimental data discretization}

In order to make the expression level measured by qPCR comparable to the discrete (i.e. 0 and 1) states that the model predicts, a discretization step was performed. As there is no specific threshold for inferring the state of the different gene products, the state was based on the relative expression of the genes. The discretization workflow is presented in Figure S1 in the Supplementary Material.

\section{Section II: In silico part}

\subsubsection{Representation of signaling network in psoriatic KCs}

A Prior Knowledge Network (PKN) encompassing the main signaling events taking place in KCs during the chronic stage of psoriasis was manually curated based on prior knowledge of the disease. The relevant to the system biological entities (nodes) and their interactions (edges) were identified by an extensive and careful assessment of the available literature and by their extraction from databases with causal molecular interactions, such as Signor (Perfetto et al. 2016). The metabolism of those eicosanoids with a reported role in psoriasis and those identified through our in vitro experiments was also added to the keratinocyte model. The rest of the curation was focused on the signaling cascades initiated by the main cytokines that characterize psoriatic lesions in the chronic stage of the disease, namely IL-17, IL-22, TNF $\alpha$, and IFN $\gamma$. Additionally, guided by in vitro observations (presented in Section 3.1), signaling mechanisms that occur downstream of $\mathrm{PGE}_{2}$ were added to the model to allow for hypothesis-driven simulations. In order to allow the exploration of the potential synergistic effect between $\mathrm{cPLA}_{2} \mathrm{a}$ inhibition and vitamin $\mathrm{D}$ analogs like calcipotriol, signaling through the Vitamin-D receptor (VDR) was included. Each signaling component was annotated by its official gene symbol, UniProt ID or ChEBI ID, while all interactions were annotated with the PubMed IDs supporting their addition to the model. 
2.2.2 The logical model of psoriatic keratinocytes

After the identification of the relevant nodes and their regulatory interactions with other nodes, the network was converted to a Boolean model and encoded in the GINsim software (Naldi et al, 2018). In Boolean models, the activity of a node (also called state) can be associated with Boolean values (i.e. 1 or 0 ). Signaling components with a value of 1 are considered active while a value of 0 is associated with inactive components. Lastly, for each node, the logical rules governing its state were specified. The logical rules aimed to capture how a node's state depends on the state and combinations of its regulators and were manually specified following the logical formalism AND, OR, and NOT. The definition of the rules was an iterative process based on the results of various model analyses and their comparison to the expected behavior described in the literature and in our in vitro observations.

The final model was analyzed using software tools developed by the CoLoMoTo consortium (http://www.colomoto.org/). The analyses explored the system from several angles aiming i) to assess its performance and validate its closeness to biological reality, ii) to use the model as a tool to understand how $\mathrm{cPLA}_{2} \mathrm{a}$ downstream lipid mediators and more specifically $\mathrm{PGE}_{2}$, together with Th17 and Th1 cytokines contribute to the different phenotypes of the disease, and iii) to explore how the targeting of $\mathrm{cPLA}_{2} \mathrm{a}$ alone or together in treatments with vitamin $\mathrm{D}$ analogs affects the system. The model analysis in full is performed and archived as a Jupyter Notebook to ensure reproducibility.

\subsubsection{Validating model performance - Stable states \& perturbations}

With the purpose of validating the performance of the model with respect to its ability to correctly predict experimental observations and its fidelity to biological reality, the stable states or attractors (comparable to cellular states, or phenotypes) of the model under various conditions were computed using the bioLQM toolkit (Naldi, 2018). The cellular states that the model can emulate under various external stimuli can be deduced from the local states of specific subsets of model components that can be used as markers. The balance between the activating and inhibiting states of markers depends on the model's response to specific inputs and dictates which phenotypes will be reached. For instance, whether a cell will undergo apoptosis does not depend on the activation of pro-apoptotic regulators, but rather on the ratio between pro-apoptotic (e.g. Bax) and anti-apoptotic proteins (e.g. BCL-2) (Khodapasand et al, 2015, 2).

The model's validation and analyses were performed in three main conditions, which are summarized below. In addition to the conditions tested in vitro (i.e. the Th17-derived IL-17 and IL-22), a simulation more representative for the psoriatic milieu, where IL-17, IL-22, TNF $\alpha$, and IFN $\gamma$ are present, was also included. Additionally, in order to elucidate the potential distinct role of the Th17- and Th1-derived cytokines, the behavior of the model in the presence of the Th1 (i.e., IFN $\gamma$ and TNF $\alpha$ ) and Th17 cytokines separately was explored. Lastly, as EP4 appears to be regulated in response to IL-17 and IL-22 (experimental observations presented in Section 3.1), the receptor was encoded to be active in the simulations.

The three psoriatic conditions that were chosen for the simulations are summarized below:

- TH17: Only Th17 cytokines are present, and EP4 is active.

- TH1: Only Th1 cytokines are present, and EP4 is active.

- ALL: All Th17 and Th1 cytokines are present, and EP4 is active. 
As a next step, the behavior of the system under different chemical perturbations was simulated. In all of the three above conditions (i.e. TH17, TH1, ALL), three perturbations were performed:

- AVX: Inhibition of cPLA2

- CAL: Activation of VDR receptor

- COMBO: Combination of AVX and CAL

Regulatory circuits and their functionality were analyzed in order to investigate the existence of multiple attractors and/or cyclic attractors. Functional positive circuits (or positive feedback loops) can give rise to multiple attractors, while functional negative circuits (or negative feedback loops) can possibly result in cyclic attractors (Thieffry, 2007).

\subsubsection{Assessing the system's state evolution over "time" through stochastic simulations}

The probabilistic interpretation of node states, phenotypes, and their potential transient behavior were explored with stochastic simulations using MaBoSS (Stoll et al, 2017). Additionally, the 'time'-dependent evolution of the markers' states together with the probability of reaching a phenotype were analyzed. MaBoSS allows the calculation of phenotype probabilities when model variables, such as node states or inputs, are altered, allowing the comparison of how the behavior of a system depends on those changes. Stochastic simulations were performed for three conditions; with only Th17 cytokines, with only Th1 cytokines, and with all cytokines present. The analysis was performed to identify whether the evolution of the system could vary depending on the inputs, or whether the system would reach a psoriatic state with either of the two sets of cytokines. In order to ensure that the whole state space was explored, a high number of trajectories (10.000) was chosen. The fraction of instances (from a total of 10.000) a state is occupied is taken as the probability of that state. In a cell population cells may occupy various states with different sets of entities active in each cell, which may result in a different response to the same input, depending on the specific state of that cell. For the majority of the analyses, and unless stated otherwise, the initial state of the cells was not taken into account for the simulations, and the evolution of the system, as well as the probabilities of reaching a state, were calculated while all entities, but the chosen inputs, were inactive.

2.2.5 Understanding the impact of each cytokine in the system through value propagation analysis A comparison of the impact of Th17- and Th1-derived cytokines was investigated by a value propagation study, as described in (Hernandez et al, 2020). The same analysis was also performed for each cytokine individually. The latter comparisons were conducted to identify whether cytokines produced by the same Th-cell populations have complementary or overlapping actions and to compare the influence of each cytokine on its own. In value propagation analysis, a value of 1 is assigned to a node of interest (emulating its activity), in our case the different cytokine receptors of the model, and propagated through the model. This allows the identification of those nodes that are bound to be active or inactive in one of the two compared conditions or in both, and, therefore, aids the comparison of the impact of specific inputs to the system. While setting a value of 1 to the node(s) of interest, the value of the VDR node was set to 0 in all comparisons. This ensured that the analysis would include the influence only of the disease-related nodes, as the effect of Vitamin D analogs had been encoded in the model as an input node that regulates the state of downstream nodes as any other.

\subsubsection{Identification of perturbations to restore a normal phenotype}

Finally, an analysis was performed in order to explore the complete node perturbation space (both singly and combinatorial) and identify potential perturbations (activating or inhibiting) that would drive the diseased system in a preferred direction and drug targets that could potentially be explored as treatment 
options. This was performed as a mutation analysis with the Pint tool (Paulevé, 2017), which identifies perturbations that will prevent the activation of the proliferation and inflammatory markers, and the inactivation of apoptotic and differentiation markers, essentially signifying the return to a normal $\mathrm{KC}$ phenotype. Pint provided a list of combinations for each of the markers. The combinations were then analyzed and ranked with respect to the frequency that a single or combinatorial perturbation was proposed. It should be noted that the tool might propose combinations that are in fact redundant, meaning that the addition of a second perturbation will have the exact same impact as the individual one. The presented list in the results is curated to include only those perturbations with a distinct impact in the system.

\section{Data Availability}

The model and the notebook containing its full analysis are publicly available at https://github.com/Eirinits/psoKC model and at the GINsim model repository (http://ginsim.org).

\section{Results}

We set out to develop an in silico model of psoriatic KC that could be used to describe the development of cytokine-dependent phenotypes of psoriatic KCs and the therapeutic mechanism of action of $\mathrm{cPLA}_{2} \alpha$ inhibitors alone and in combination with other anti-inflammatory agents. The construction of the logical model started with describing the wide range of experimental observations regarding the eicosanoid signaling taking place in Th17-cytokine-stimulated KCs that the model should reconcile. Prior knowledge on the system together with experimental observations was integrated into a regulatory network which was then used to simulate the response of KCs to external stimuli and predict their effect on the psoriatic environment with respect to the secretion of ligands and intercellular-acting stimuli. A detailed description of the workflow is provided in the Supplementary information.

\subsection{Th17 cytokines regulate $\mathrm{KC}$ differentiation and prostaglandin E2 release in vitro}

In vitro models of psoriasis can be created by exposing monolayers or $3 \mathrm{D}$ skin-equivalent cultures to psoriatic cytokines (Desmet et al, 2017). We have previously used the immortalized KC cell line, HaCaT, in $3 \mathrm{D}$ skin-equivalent cultures to document the role of $\mathrm{CPLA}_{2} \alpha$ in $\mathrm{KC}$ proliferation (Ashcroft et al, 2020). These cells express both $\mathrm{cPLA}_{2} \alpha$ and $\mathrm{sPLA}_{2}$, and have been used to study the regulation of eicosanoid production by Th1 cytokines in the skin (Sjursen et al, 2000; Thommesen et al, 1998). HaCaT cells are also known to respond to Th17 cytokines (Woo et al, 2017) but the role of $\mathrm{cPLA}_{2} \alpha$ in mediating the effects of Th17 cytokines in KCs is not known.

To investigate the role of $\mathrm{cPLA}_{2} \alpha$ in Th17-dependent signaling in $\mathrm{KCs}$ we used air-exposed 3D cultures of HaCaT grown in the presence of a combination of IL-17 and IL-22 (from hereon referred to as Th17 cytokines). Immunohistochemical staining of fixed, paraffin-embedded cultures was used to quantify proliferating and differentiating cells, using Ki67 and cytokeratin (CK) 10 positivity respectively. Eicosanoid release was measured by ELISA. The control cultures were 140-180 $\mu$ m thick, typically comprising 4-5 cell layers; approximately $20 \%$ of the cells were Ki67 positive, consistent with being in a proliferative state, while approximately $30 \%$ were CK10 positive, indicating early differentiation. Treatment with Th17 cytokines did not affect the thickness of these cultures but, consistent with several studies (Rabeony et al, 2014; Nograles et al, 2008; Boniface et al, 2005; Pfaff et al, 2017), affected differentiation, as evidenced by the loss of CK10 expression. The Th17-treated cultures also had significantly reduced Ki67 positivity indicating lower proliferation, which is inconsistent with the 
hyperproliferative state of the psoriatic epidermis. Treatment with either AVX001, CAL or COMBO had no effect on the thickness, CK10 or Ki67 positivity of the Th17-treated cultures (Fig 1A-B).

Increased levels of the prostaglandin $\mathrm{PGE}_{2}$ were measured in the supernatants from cultures treated with Th17 cytokines, while leukotriene B4 (LTB4) and 12s-HETE, were not affected. Suppression of cPLA $2 \alpha$ activity using the cPLA2 $\alpha$ inhibitor AVX001 (Huwiler et al, 2012, 00) reduced the $\mathrm{PGE}_{2}$ levels implicating the activation of the enzyme in this response. Similarly, the use of the vitamin D analog and topical antipsoriatic drug calcipotriol $(10 \mathrm{nM})$ reduced the $\mathrm{PGE}_{2}$ levels, and when we combined both compounds (COMBO) $\mathrm{PGE}_{2}$ levels were similar to the unstimulated controls (Fig 1C).

\subsection{Regulation of $\mathrm{PGE}_{2}$ biosynthesis and signaling by Th17 cytokines in KCs in vitro}

The biosynthesis of $\mathrm{PGE}_{2}$ results from the co-ordinated regulation of several enzymes; free arachidonic acid (AA) released as a result of phospholipase $\mathrm{A}_{2}$ activity is metabolized to $\mathrm{PGH}_{2}$ by the activity of cyclooxygenases, Cox-1 (PTGS1) and Cox-2 (PTGS2), which is further metabolized to $\mathrm{PGE}_{2}$ by prostaglandin synthases (PTGES1, PTGES2, PTGES3). Intracellular $\mathrm{PGE}_{2}$ can be oxidized to an inactive 15-keto form by the enzyme 15-prostaglandin dehydrogenase (PGDH) or released into the extracellular space where it is an autocrine or paracrine ligand for the eicosanoid-prostaglandin (EP) family of receptors (PTGER1-4) residing at the cell surface. While, psoriatic lesional skin has been shown to overexpress enzymes involved in $\mathrm{PGE}_{2}$ biosynthesis and under-express $15-P G D H$ (Lee et al, 2019) the separation between regulation of the $\mathrm{PGE}_{2}$ biosynthesis pathway in KCs versus infiltrating immune cells was not made.

In order to investigate the mechanism of regulation of $\mathrm{PGE}_{2}$ production by Th17 cytokines for integration into the computational model, we measured the expression of genes involved in $\mathrm{PGE}_{2}$ biosynthesis and signaling. Total RNA was extracted from the HaCaT cultures treated with Th17, in the absence or presence of AVX001, calcipotriol, or a combination of AVX001 and calcipotriol (COMBO). The expression of PLA2G4A, PTGS1, PTGS2, PTGES1-3, 15-PGDH, four prostaglandin E receptors (PTGER1-4), and three internal reference genes (TBP, HPRT1, and GAPDH) were measured by quantitative PCR. Results are shown relative to the unstimulated control (CTRL) (Fig 1D, Fig S2). Hierarchical clustering of log-transformed values identified 3 groups characterized by upregulation, downregulation, or a lack of regulation by Th17 cytokines. PTGS2 and PTGER4 were up-regulated while PTGS1, PTGER2 and 15-PGDH were down-regulated. A trend towards up-regulation of prostaglandin E synthases was also observed, though no changes reached statistical significance at $\mathrm{p}<0.05$. With the possible exception of PTGES3, calcipotriol treatment did not reverse the effects of IL-17 on PTGS, PTGES or 15-PGDH expression, indicating suppression of $\mathrm{PGE}_{2}$ release by calcipotriol may occur via an independent mechanism. Interestingly, both calcipotriol and COMBO treatments rescued the effect of Th17 cytokines on PTGER4 and PTGER2 expression.

In summary, Th17 cytokines stimulated $\mathrm{PGE}_{2}$ release from KCs. This was dependent upon cPLA2 $\alpha$ activity and was associated with up-regulation of PTGS2 and down-regulation of 15-PGDH gene expression. Th17 additionally suppressed PTGER2 and increased PTGER4 expression. Combining AVX001 with calcipotriol completely suppressed Th-17-dependent PGE $_{2}$ release and reversed the Th17-dependent switch from PTGER2 to PTGER4 expression (summarised in Fig 1E). These findings were integrated into the in silico keratinocyte model as Th17 cytokine-dependent activation of $\mathrm{cPLA}_{2} \mathrm{a} / \mathrm{COX}-2 / \mathrm{EP} 4$ signaling as described below. 
A. (i) CTRL

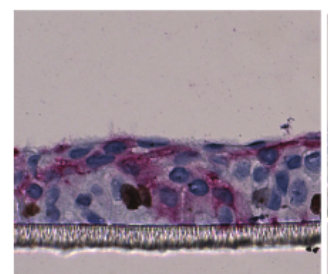

(ii) Vehicle

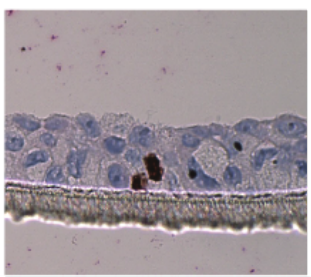

(iii) AVX001

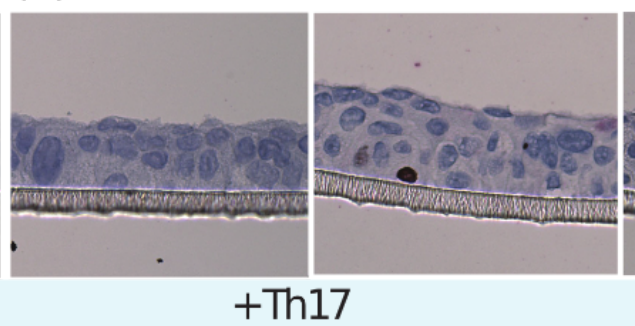

(v) Combo

B. (i)

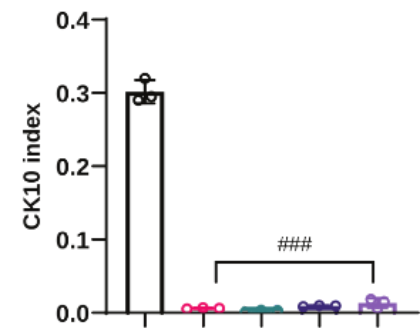

C. (i)

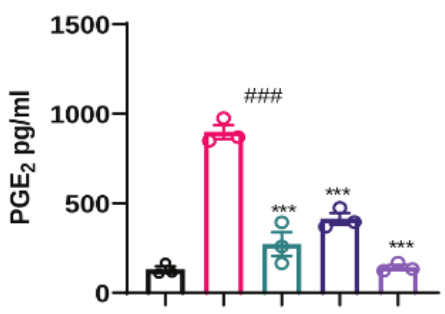

D.

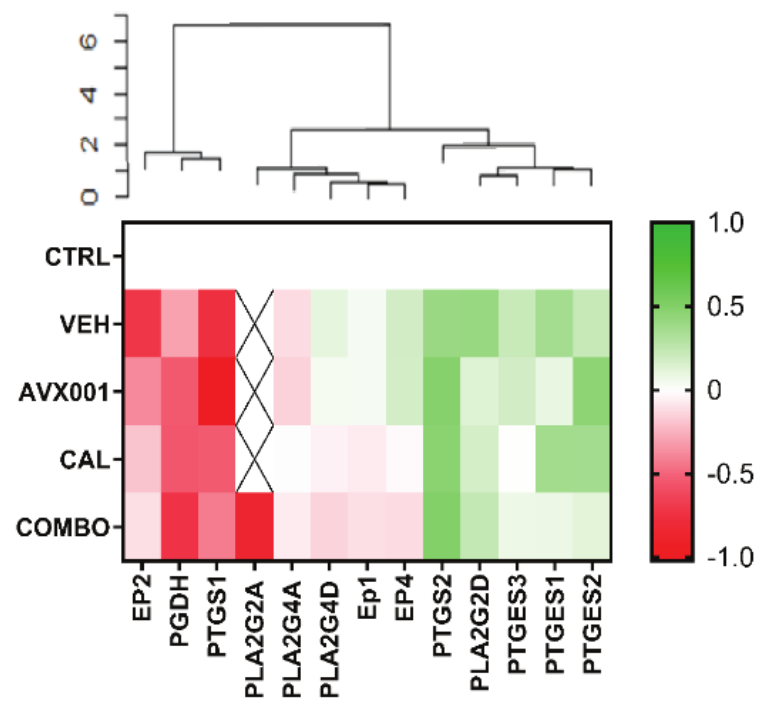

(ii)

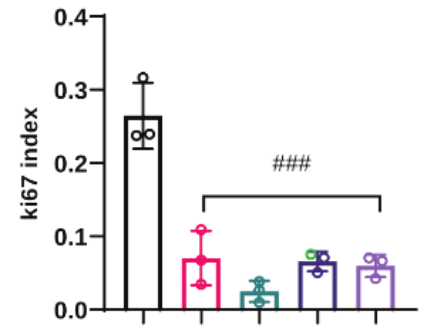

(ii)

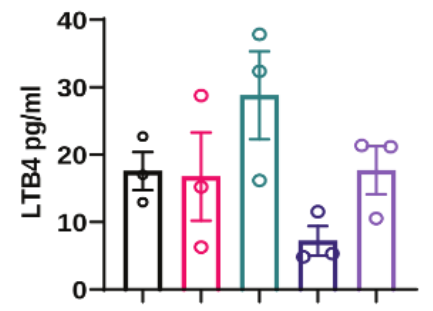

(iii)

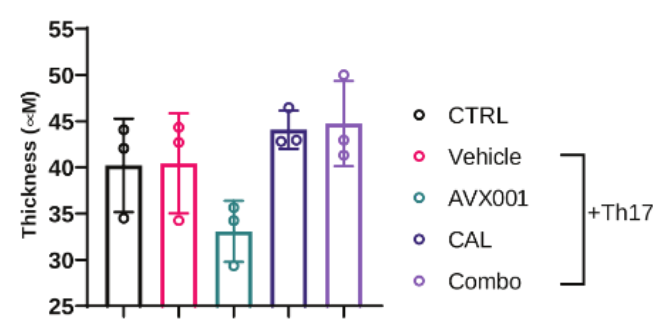

(iii)

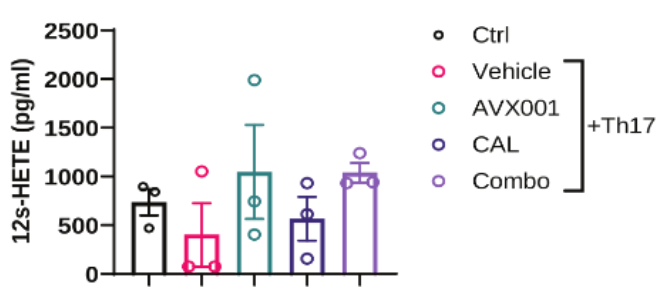

E.

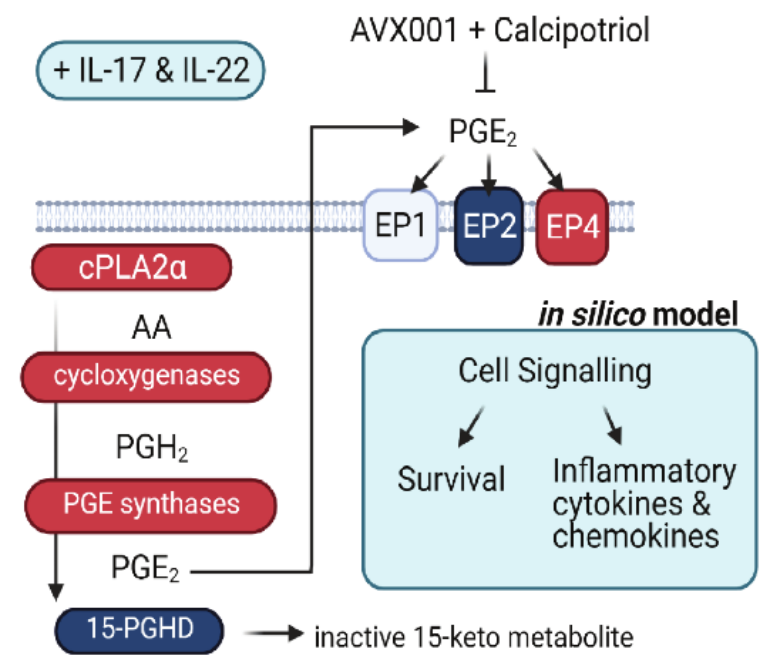


Figure 1. Regulation of cPLA2/PGE/EP4 signaling by Th17 cytokines. A. Representative images showing immunostaining with anti-Ki-67 (DAB+) and anti-cytokeratin 10 (Permanent Red) antibodies B. Quantification of (i) the proportion of CK10 positive cells (ii) the proportion of proliferating cells and (iii) the thickness of the cultures. The data shown are the mean \pm SEM for three replicates. C. Eicosanoid levels measured by ELISA for (i) $\mathrm{PGE}_{2}$, (ii) 12 s-HETE and (iii) $\mathrm{LTB}_{4}$. Data shown are the mean $\pm \mathrm{SEM}$ of three replicates, measured in duplicate. D. Heatmap of $\log _{10}$ fold changes in expression relative to unstimulated controls (CTRL) for genes involved in $\mathrm{PGE}_{2}$ synthesis, degradation and signaling. Data is the mean of three replicates. $\mathrm{X}$ indicates that expression was undetectable E. Schematic summary showing how Th17 cytokines could regulate $\mathrm{PGE}_{2}$ release and activate downstream signaling events via the EP4 receptor. Components in red were up-regulated and components in blue were down-regulated in cultures treated with Th17 cytokines.

\subsection{The logical model of psoriatic KCs}

To recapitulate all these observations in a consistent regulatory model that captures as much as possible the deregulatory events leading to psoriasis we focused on the representation of the KCs as responders to Th cell-derived cytokines. The psoriatic keratinocyte (psoKC) model is presented in Fig $\mathbf{2}$. The model aims to integrate the available knowledge on regulatory interactions which take place during the chronic stages of psoriasis, including the newly described regulation of $\mathrm{PGE}_{2}$ signaling. The model contains 90 biological entities (nodes) and 176 regulatory interactions (edges) and can be stimulated by the activation of the receptors recognizing the main psoriatic cytokines, namely IL-17, IL-22, TNF $\alpha$, and IFN $\gamma$, and/or $\mathrm{PGE}_{2}$ (EP) receptors. It is important to highlight that the regulatory mechanism of $\mathrm{CPLA}_{2} \mathrm{a}$ in the system is mainly designed to be able to assess the effect of $\mathrm{PGE}_{2}$ through the EP receptors. This effect is encoded in a way that the activation of EP receptors is an input and not directly activated by the PGE2 node in the model. To account for the autocrine effects of $\mathrm{PGE}_{2}$ in $\mathrm{KCs}$, the $\mathrm{EP}$ receptors were set to be active in simulations where $\mathrm{CPLA}_{2} \mathrm{a}$ activity is uninhibited. In order to distinguish the EP receptor nodes from their genes, which are activated by different transcription factors downstream in the model, a suffix $g$ was added to the nodes that represent their respective genes.

The model is able to describe the three aberrant phenotypes of $\mathrm{KC}$ in psoriasis: hyperproliferation, resistance to apoptosis, and aberrant differentiation. The model furthermore covers the KC's immunostimulatory states, as manifested by the production of cytokines, chemokines, and AMPs (antimicrobial peptides) that activate, recruit and maintain immune cell populations that contribute to the psoriatic phenotype. The phenotypes and state of the system can be inferred from the state (ON or OFF) of selected sets of markers that are characteristic of each specific cellular behavior. The marker nodes were defined and selected as those entities which are included in the transcriptional signatures of the psoriatic cytokines on KCs and associated with one or more phenotypes that the model aimed to represent. The node-cellular state association can be seen in Table $\mathbf{1 .}$ 


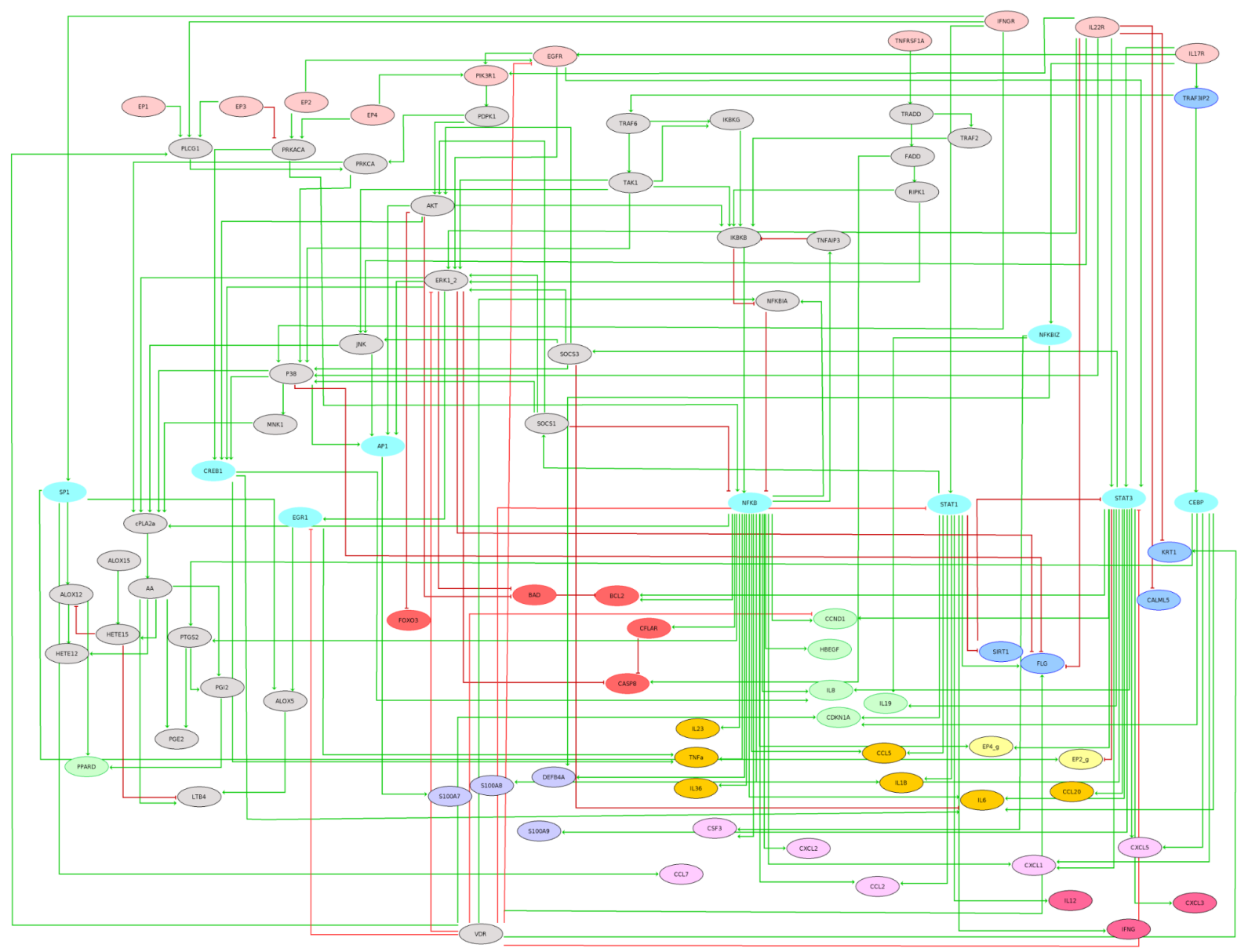

\section{Node legend}

Receptors

Signaling Proteins

Transcription Factors
Inflammation

Th1

Th17
Apoptosis

Differentiation

Proliferation \& Survival

Figure 2. The logical model of psoriatic keratinocytes. The node color depicts their functional role, the phenotype they promote, or the immune cell types they act on. Green lines represent activating interactions and red lines represent inhibitory interactions. 
Table 1. Marker-nodes which were used to define the model's physiological state and their associated phenotypes and processes. Nodes are named by their HGNC symbol for genes and proteins and their ChEBI ID for eicosanoids. Nodes in red cells are inhibiting the phenotype associated with their respective column. Nodes with an asterisk $(*)$ were experimentally tested.

\begin{tabular}{|c|c|c|c|c|c|c|c|}
\hline \multicolumn{3}{|c|}{ Keratinocyte markers } & \multicolumn{5}{|c|}{ Inflammatory \& immunostimulatory markers } \\
\hline $\begin{array}{c}\text { Proliferation } \\
\& \text { Survival }\end{array}$ & Differentiation & Apoptosis & Inflammation & Th1 & Th17 & $\begin{array}{c}\text { Other } \\
\text { immune } \\
\text { cells }\end{array}$ & PGE2-related \\
\hline CCND1* & FLG* & BAD* & S100A7 & IFNG* & IL-1B* & IL6* & PTGER2_g* \\
\hline $\begin{array}{l}\text { CXCL8/ } \\
\text { IL8* }\end{array}$ & KRT1* & CASP8 & S100A8 & CXCL3 & TNFA* & CXCL1* & PTGER4_g* \\
\hline PGE2* & CDKN1A* & BCL2* & S100A9 & IL12 & CCL2* & CCL7 & \\
\hline IL19 ** & SIRT1 & CFLAR & DEFB4A & & CCL5* & CXCL2 & \\
\hline 12-HETE & CALML5 & & & & CCL20 & CXCL5 & \\
\hline PPARD & IL36 & & & & IL23 & LTB4 & \\
\hline HBEGF & TRAF3IP2 & & & & IL36 & CSF3 & \\
\hline \multicolumn{8}{|l|}{ CDKN1A } \\
\hline SIRT1 & & & & & & & \\
\hline
\end{tabular}

3.4 Integration of experimental observations into the model \& validation with in vitro results Our experimental observations suggest that $\mathrm{cPLA}_{2} \mathrm{a} / \mathrm{PGE}_{2} / \mathrm{EP} 4$ signaling is active in response to Th17 cytokine in KCs. The potential involvement of this pathway in the development of psoriasis was tested in model simulations by comparing the states of phenotypic marker nodes when EP4 was active and inactive. In conditions where EP4 was inactive, CFLAR, CREB1, IL-8, CSF3, DEFB4A, IL-23, IL-36 genes were also predicted to be inactive. However, upon the activation of EP4 in the simulations, the states of the aforementioned entities were corroborating the available literature on the state of these entities in psoriasis.

In order to further investigate the role of $\mathrm{CPLA}_{2} \mathrm{a} / \mathrm{PGE}_{2} / \mathrm{EP} 4$ signaling experimentally, the expression of 17 phenotypic marker genes shown in Table I was measured by qPCR. Th17 up-regulated the expression of pro-inflammatory cytokines (IL6,IL1 $\beta, T N F-\alpha, I L-8, C C L 2, C X C L 2)$ and down-regulated genes associated with differentiation ( $F L G, K R T 1)$ and apoptosis $(B A D)$ (Fig 3 and Fig S3). Inhibition of the $\mathrm{CPLA}_{2} \mathrm{a}$ using AVX001 or treatment with calcipotriol alone suppressed the induction of $C C L 2$ and prevented the down-regulation of $B A D$; calcipotriol additionally caused a partial rescue of the loss of KRT1 expression. Combining AVX001 with calcipotriol inhibited more of the proinflammatory markers (CCL2, $I L-6$, and $I L-8$ ). To compare these experimental results to the Boolean states of the corresponding nodes in the model, the gene expression data were discretized to 1s and 0s (As described in Fig S4). For 
59 of the 68 experimental observations, the model predictions were in agreement (Fig 3). Interestingly, the nine observed discrepancies were seen only in perturbed simulations, with four of these nine discrepancies occurring with the use of the $\mathrm{CPLA}_{2} \mathrm{a}$ inhibitor alone.

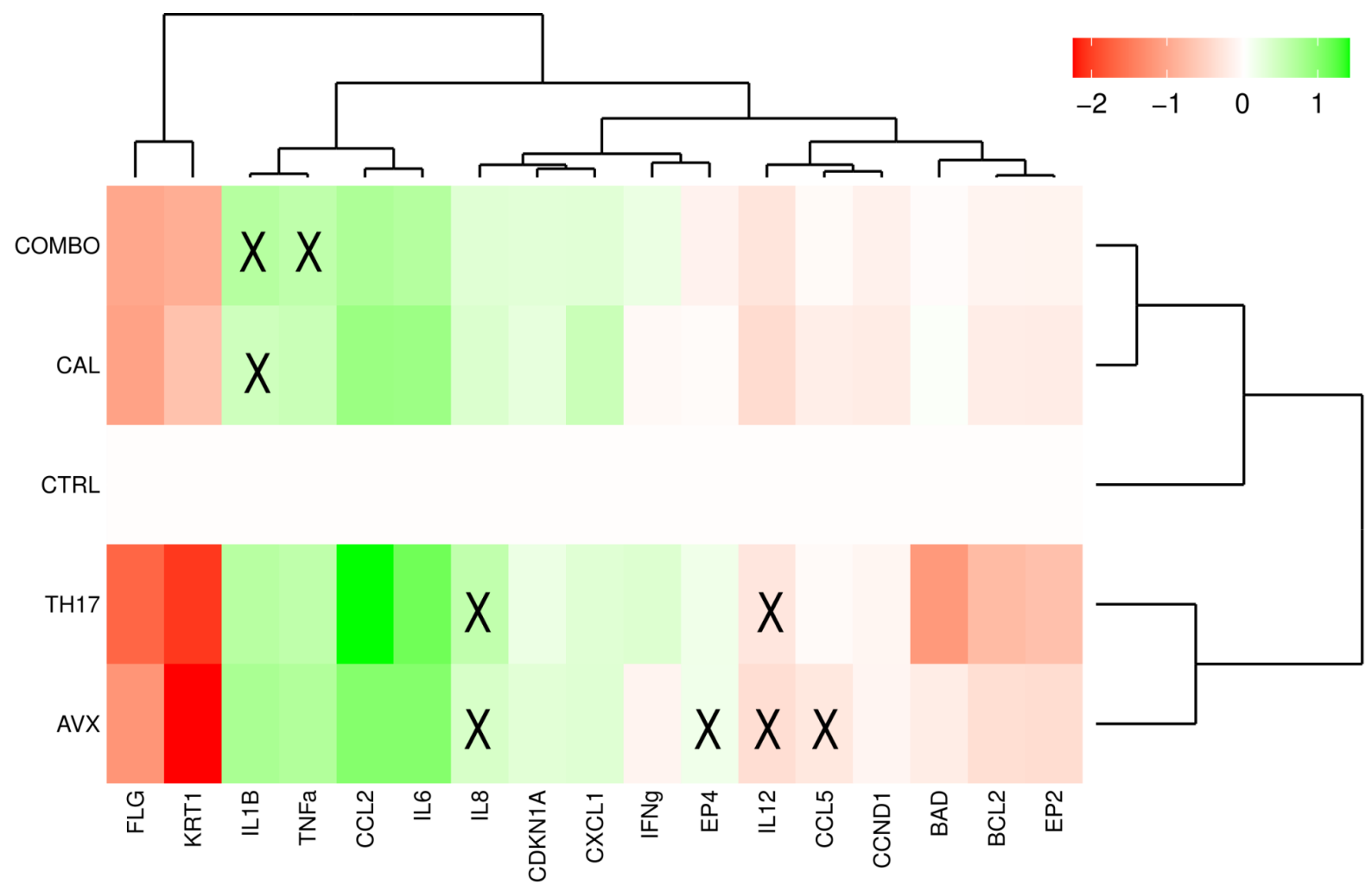

Figure 3. Comparison of experimental observations with node states predicted by the computational model. Heatmap of $\log _{10}$ fold change in gene expression relative to CTRL as determined by qPCR. HaCaT 3D cultures were treated with Th17 cytokines (TH17) in the presence of AVX001 (AVX), calcipotriol (CAL), or a combination of AVX001 and calcipotriol (COMBO). Rows and columns are hierarchically clustered. X represents data points where the results from the computational model do not agree with the experimental observation.

\subsection{Predicting keratinocyte behavior upon different stimuli and treatments}

Encouraged by the apparent value that the logical model has in predicting verifiable experimental results, we performed a series of model simulations in an effort to gain further insight into the regulatory mechanisms underlying psoriasis. As previously described, Th17 cells and their produced cytokines play a critical role in psoriasis. However, it has been suggested that different Th-cells types are dominating and controlling the inflammatory process during the various stages of the disease (Furiati et al, 2019), but in reality, Th1, Th17, and Th22 cells all together contribute to its development (Diani et al., 2016). Therefore, the downstream simulations and experimentations were focused on gaining a better understanding of how different sets of cytokines, and by extension, different sets of Th cells, affect the behavior of KCs, how this behavior changes when all Th1 and Th17 cytokines are present and, lastly, how $\mathrm{PGE}_{2}$-regulated signaling is integrated into the system.

The response of KCs to stimuli from Th1 (i.e. IFN $\gamma$ and TNF $\alpha$ ), Th17 (i.e. IL-17 and IL-22), and Th1/Th17 combined was simulated. For the same conditions, the effect of chemical perturbations with $\mathrm{cPLA}_{2} \mathrm{a}$ inhibitors, vitamin D analogs, or a combination of the two was simulated. The simulation results are presented as a series of active and inactive entities for each condition in Fig 4. All perturbed-system 
simulations were able to reach a single stable state, a phenomenon that can be attributed to the absence of positive regulatory circuits (Thieffry, 2007), as identified by a functional circuit analysis performed on the logical model.

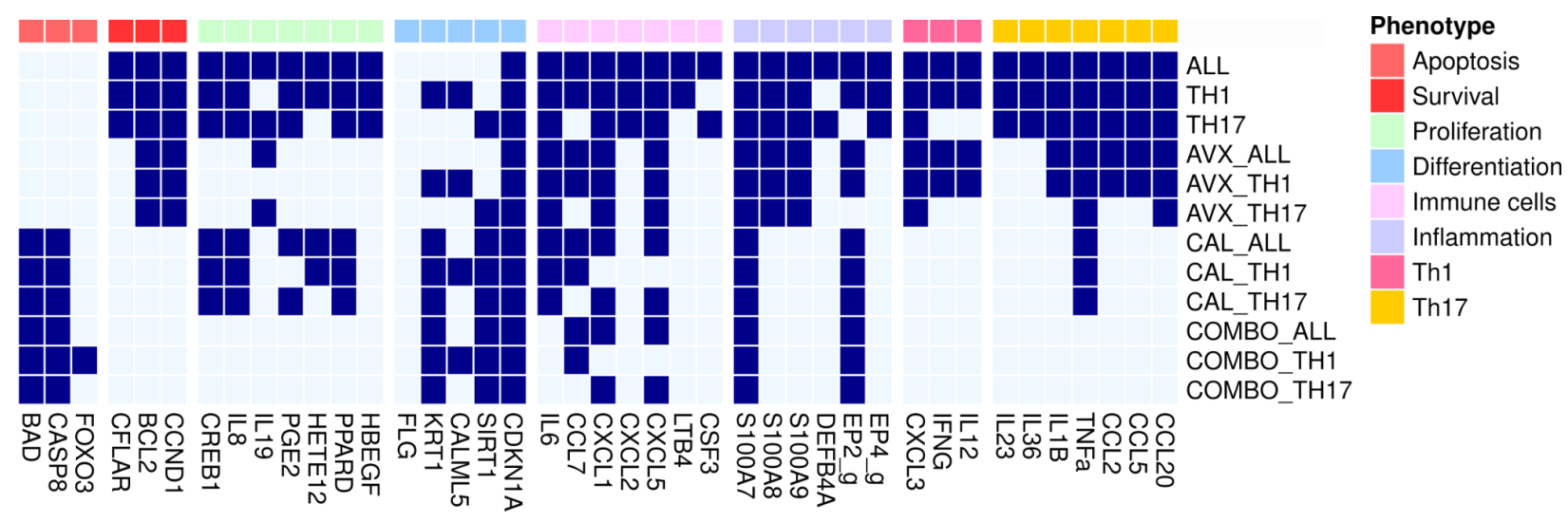

Figure 4. Heatmap of system's perturbations.

The heatmap depicts the set of active and inactive marker nodes, grouped by their associated phenotype, in each simulated condition. Light blue denotes inactive entities while dark blue denotes active entities, the number of which can be taken as a measure of compliance with specific phenotypes.

As displayed in the first three rows of the heatmap, which represent unperturbed conditions, the model predicts that all cytokines contribute to the maintenance and amplification of the positive feedback loop between KCs and Th cells. However, it is only when all four cytokines are present (ALL condition in Fig 4) that all immunomodulatory markers are activated. The significant overlap between the Th1 and Th17 conditions could indicate that the two sets of inputs act synergistically rather than complementary, as similar observations were made when analyzing the synergistic effect of IL-17, TNF $\alpha$, and IFN $\gamma$ (Chiricozzi et al, 2011). Interestingly, the inhibition of $\mathrm{CPLA}_{2}$ a (rows 4-6) mainly affects proliferation markers and only some inflammatory and immune cell markers. These results agree with previous studies of the role of PLA2 enzymes and their downstream products in psoriasis (Ashcroft et al, 2020) and skin biology in general (Murakami et al, 2018). Moreover, it is worth noting that the targeting of $\operatorname{cPLA}_{2} \mathrm{a}$ appears to mainly act on the Th17 cell-related markers, but not on the Th1 markers. However, as the model is mainly focusing on the role of $\mathrm{PGE}_{2}$ in the system, it is possible that other $\mathrm{CPLA}_{2} \mathrm{a}$ downstream products affect Th1 cell markers, but that their associated mechanisms are not depicted in the current version of the model.

When comparing the effect of $\mathrm{cPLA}_{2} \mathrm{a}$ inhibition with the effect of calcipotriol (rows 4 - 6 and 7 - 9 in Fig 4), it is evident that both drugs have a distinct mechanism of action, as calcipotriol appears to affect a different set of markers than $\mathrm{cPLA}_{2} \mathrm{a}$. More specifically, calcipotriol affects mostly differentiation and apoptosis, while $\mathrm{cPLA}_{2} \mathrm{a}$ appears to mostly affect proliferation. Calcipotriol is able to have both an antiproliferative (Kristl et al, 2008; Liang et al, 2017) and a pro-apoptotic effect (Huang et al, 2019; Tiberio et al, 2009) in psoriatic KCs. Apoptosis is subject to an elaborate regulation and controlled by the ratio of pro- to anti-apoptotic regulators, meaning that both types of regulators can be active at the same time (Jan \& Chaudhry, 2019). As seen in Fig 4, calcipotriol appears to shift the balance towards pro-apoptotic regulators and appears to promote apoptosis in the system, regardless of the input conditions. At the same time, proliferation-promoting eicosanoids, such as $\mathrm{PGE}_{2}$ and $12 \mathrm{~s}-\mathrm{HETE}$, are predicted to still be produced, unless $\mathrm{CPLA}_{2} \mathrm{a}$ is inhibited. The same behavior has been reported in various dying cells, where $\mathrm{PGE}_{2}$ is released as a Damage-Associated Molecular Pattern (Hangai et al, 2016). 
Keratinocyte differentiation is also rescued by calcipotriol, as indicated by the states of the differentiation markers. Interestingly, terminal differentiation of KCs shares many similarities with apoptotic mechanisms (Terskikh \& Vasil'ev, 2005) as can be seen in the coordinated change of both sets of markers.

Finally, as seen in the COMBO simulations, a distinct change of the markers' behavior is evident, both in the $\mathrm{KC}$ cell fate and the suggested effect of the $\mathrm{KC}$ cell on immune cell behavior. While we cannot directly quantify the effect of the different drugs and their combination, it is clear that the combination of the two drugs results in what appear to be additive changes in the system. CPLA 2 a inhibitor and calcipotriol together inhibit all endogenous and exogenous markers that would promote proliferation and survival. Furthermore, the recruitment of immune cells appears significantly impaired (absence of chemoattractants and immunostimulating cytokines), while primary proinflammatory cytokine TNF $\alpha$ is also inhibited when both drugs are used. The effect on differentiation, however, can be attributed solely to calcipotriol, as the additional $\mathrm{CPLA}_{2} \mathrm{a}$ inhibition in the combo treatment has no effect on these phenotype markers.

3.6 Evolution of the regulatory system through "time", and phenotype probabilities

While a stable state analysis provides great insights into which stable states a system can occupy, intuitively analogous with cellular phenotypes, stochastic simulations approximate the observation of transient behaviors and the evolution of the states of the nodes until the model reaches those stable states.

As observed in our in vitro experiments and reported in the literature (Ekman et al, 2019; Pfaff et al, 2017; Boniface et al, 2005), stimulation by Th17 cytokines, and mainly IL-22, completely suppresses differentiation and apoptosis in KCs, with survival and anti-differentiation markers being active, and differentiation markers inactive with a $99.9 \%$ probability in the stochastic simulations. The promotion of an antiapoptotic phenotype by Th1 cytokines was also confirmed by stochastic simulations, where an anti-apoptotic phenotype was reached with $100 \%$ probability (Fig S5). Nevertheless, other studies have shown that under certain conditions, TNF $\alpha$ and IFN $\gamma$ can induce apoptosis in KCs (Viard-Leveugle et al, 2013; Reinartz et al, 1996). To see if this behavior was dependent on the state of specific components of the system, simulations with active TNF $\alpha$ and IFN $\gamma$ were performed with an exhaustive series of initial conditions where all entities had a 50\% chance of being active. While the system was now able to reach several states with different sets of markers being active each time, the states could be separated into apoptosis and survival. By analyzing the difference between those states, two proteins seemed to be determinants for whether apoptosis would be reached; SOCS1 and SOCS3. Cell populations with active SOCS1 and SOCS3 were able to escape apoptosis (Fig 5). Both proteins were found overexpressed in psoriatic skin when compared to normal skin (Federici et al., 2002), and their activity may be contributing to the resistance of psoriatic KCs to cytokine-induced apoptosis, via a mechanism involving the activation of PI3K/AKT and NFKB pathways (Madonna et al, 2012). Simulations of the dynamic interplay and temporal evolution of the states pro-apoptotic and anti-apoptotic markers revealed that the combinatorial treatment, which inhibits the survival markers, renders pro-apoptotic marker activity unconstrained so that they reach a final active stable state (Supplementary Fig S6). 


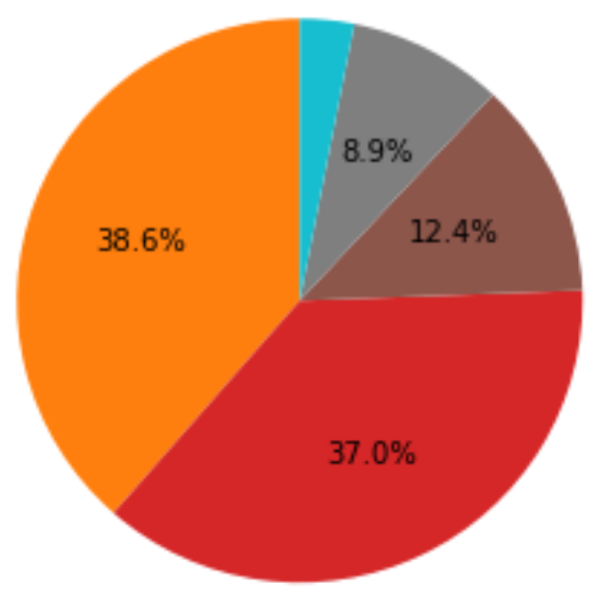

CREB1 - CASP8 -- BAD

SOCS3 -- SOCS1 -- CREB1 -- CFLAR -- BCL2 -- CCND1

- SOCS3 - SOCS1 - BCL2 -- CCND1

CASP8 - BAD

CASP8 - BAD -- FOXO3
Figure 5: Stable state probability pie chart.

Probabilities of stable states after the stimulation with IFN $\gamma$ and TNF $\alpha$ in an unsynchronized population of cells as calculated by stochastic simulations. Only selected survival and apoptosis markers are shown.

3.7 Understanding the impact of each cytokine in the system

To explore in more depth the mechanistic regulation of different inputs that leads to the distinct phenotypes identified by the previous analysis, a 'value propagation' analysis was performed allowing to trace in detail the effects of various inputs through the regulatory graph. An analysis of these effects can be used to identify which specific entities are bound to get activated or inactivated by which specific inputs. A comparison of the effects of the inputs should then indicate whether one or more cytokines that derive from the same Th-cell population are sufficient to induce characteristic psoriatic phenotypes or whether it is rather the integration of the stimuli from different Th-cell populations that results in these phenotypes.

The analysis revealed that stimulation with the Th1-derived cytokines impacted more phenotypic markers than stimulation with IL-17 and IL-22 (see Table 2 and Fig S7 in Supp Mat), as also seen in the stable state analysis. This result agrees with transcriptomic studies that have shown a dominance of Th1 and, more specifically, of IFN $\gamma$ signature expression profiles in psoriatic lesions (Albanesi et al, 2018). However, the transcription of genes of key inflammatory mediators, such as S100 antimicrobial peptides, IL-6, and TNF $\alpha$, which act synergistically to maintain an inflammatory response in psoriasis, can be activated by both sets of cytokines. The effects of the combination of IL-17 and IL-22 on differentiation and proliferation in the system that was observed in the stable state analysis (see Fig 4) and in vitro experiments (see Fig 1) are confirmed by the value propagation study, where their simulation primarily fixes the activities of differentiation and proliferation markers to states that are associated with a repressed differentiation phenotype. Conversely, IFN $\gamma$ and TNF $\alpha$ mainly fix the state of a group of nodes that is associated with Th cell maintenance. At the same time, all cytokines together lock inflammatory nodes in their active state. 
Table 2. Impact of cytokines in keratinocyte phenotypes and physiological states as identified by a value propagation analysis. The number of nodes whose activity is fixed upon simulations by A) Only IL-17 and IL-22 B) Only TNF $\alpha$ and IFN $\gamma$ C) All four cytokines. The involvement of the nodes in processes is indicated in the different columns.

\begin{tabular}{|c|c|c|c|c|c|c|c|c|c|}
\hline Fixed by.. & $\begin{array}{c}\text { All } \\
\text { nodes }\end{array}$ & $\begin{array}{l}\text { Proliferatio } \\
\text { n/ Survival }\end{array}$ & Differentiation & Apoptosis & Th1 & Th17 & $\begin{array}{l}\text { Other } \\
\text { immune } \\
\text { cells }\end{array}$ & Inflammation & $\begin{array}{c}\text { Eicosanoid } \\
\text { signaling }\end{array}$ \\
\hline $\begin{array}{l}\text { IL-17 \& } \\
\text { IL-22 }\end{array}$ & 12 & 1 & 4 & - & - & - & - & - & 3 \\
\hline $\begin{array}{l}\text { TNF } \alpha \& \\
\text { IFN } \gamma\end{array}$ & 20 & - & 1 & - & 2 & 3 & 2 & - & 5 \\
\hline $\begin{array}{l}\text { IL-17, IL-22, } \\
\text { TNF } \alpha \& \\
\text { IFN } \gamma\end{array}$ & 38 & 4 & 1 & 3 & 1 & 3 & 2 & 4 & 5 \\
\hline
\end{tabular}

As cPLA $\mathrm{A}_{2} \mathrm{a}$ is of special interest as a psoriatic drug target, cytokine effects on eicosanoid production were analyzed in more detail. While all cytokines appear to be able to activate the phospholipase, the inducible enzyme COX-2, which catalyzes the production of PGE2, is activated only downstream of IL-17 and IL-22, corroborating our in vitro observations. Conversely, TNF $\alpha$ and IFN $\gamma$ induced the expression of LTB4 and 12-HETE by regulating their respective enzymes. Both $\mathrm{LTB}_{4}$ and 12-HETE are reported to play a role in the pathogenesis and development of the disease and have chemotactic properties (Nicolaou, 2013), again confirming the immunostimulatory action of Th1 cytokines. Lastly, 15-HETE, an eicosanoid with anti-inflammatory properties (Nicolaou, 2013), is found to be inactivated in all simulations and value propagations.

A comparison of the distinct effects of IL-17 and IL-22 revealed a greater overall impact of IL-17 on the regulation of both signaling proteins and markers (Fig S7), an observation that also finds support in the literature (Nograles et al, 2008; Rabeony et al, 2014). Nograles et al. also reported that IL-22 but not IL-17 regulated terminal differentiation markers of KCs (i.e. CALML5, KRT1, and FLG). Indeed, upon activation of IL-22, CALML5 and KRT1 are fixed in an inactive state, while proliferation and anti-differentiation markers (i.e IL-6 and IL-29) are active. However, a clear distinction of their role was not observed in our model analysis since both cytokines appear to have an impact on the immunostimulatory markers. In the comparative value propagation between Th1 cytokines (Fig S8), it was found that while IFN $\gamma$ dominates the regulation of immunostimulatory markers, TNF $\alpha$ has a more limited impact.

3.8 Model-based analyses to assess possibilities for the treatment of psoriasis

The availability of a logical model representation of psoriatic KCs allows a model analysis that supports an exploration of the possible perturbation space to search for model nodes that may serve as potential drug targets in treatments that could restore a normal phenotype. A full analysis of the perturbation space was performed, with each entity being considered a potential drug target, to allow the identification of novel entities whose targeting should be further explored. The activating or inhibiting, single or combinatorial perturbations that prevent the activation of the proliferation and inflammatory markers, and 
the inactivation of apoptotic and differentiation markers, are presented in Table 3. The complete lists of perturbations are available in the accompanying Jupyter Notebook.

Table 3. Frequency of perturbations that prevent the system from reaching any of the dysregulated phenotypes in psoriasis. Single or combinatorial, activating or inhibiting perturbations as predicted by PINT.

\begin{tabular}{|c|c|c|}
\hline Perturbation A & Perturbation B & Frequency \\
\hline VDR : 1 & - & 10 \\
\hline EP4 : 0 & - & \\
\hline EP4 : 0 & EP2 : 0 & 9 \\
\hline PRKACA : 0 & - & \\
\hline NFKBIA : 1 & - & \\
\hline NFKB: 0 & - & 8 \\
\hline IKBKB: 0 & - & \\
\hline STAT3 : 0 & - & 6 \\
\hline IFNGR : 0 & - & 5 \\
\hline IL17R : 0 & - & \\
\hline cPLA2a: 0 & - & \\
\hline AA: 0 & - & \\
\hline PRKCA : 0 & - & 4 \\
\hline PLCg : 0 & PIK3R1 : 0 & \\
\hline PLCg : 0 & PDPK1 : 0 & \\
\hline
\end{tabular}

Generally, the analysis identified the targeting of key inflammatory regulators as the most impactful, especially those that are converging points of multiple pathways and receptors that appear to regulate critical entities for the behavior of the system. Vitamin D analogs such as calcipotriol have been widely used to treat moderate to severe psoriasis, with generally high effectiveness (Kim, 2010). It comes as no surprise, therefore, that the activation of VDR, the receptor activated by calcipotriol, stands out as a key node that can resolve the aberrant phenotypes represented in our model.

A second important perturbation is the inhibition of the EP4 receptor, alone or in combination with EP2, confirming the important role of $\mathrm{PGE}_{2}$ and, subsequently, of cPLA $2 \mathrm{a}$ inhibition. As endogenous $\mathrm{PGE}_{2}$ is not directly activating the EP receptors in these perturbation simulations, but that their activation is fixed in the model when setting the input for the simulations, we suggest that when Pint predicts a perturbation 
that includes EP receptors, $\mathrm{CPLA}_{2} \mathrm{a}$ inhibition could be expected to have the same, or at least similar, effect in the system. The inhibition of $\mathrm{CPLA}_{2} \mathrm{a}$ or the inhibition of AA production indeed also appears among the beneficial results, although with a lower frequency. In addition to its proposal as a potential drug target by the model, the direct inhibition of EP4 is increasingly being studied as a potential treatment option in cancer, with its inhibition showing promising results alone or combined with well-established treatments (Yamamoto et al, 2020; Konya et al, 2013). Based on our model's predictions, and experimental observations, its inhibition in systems where inflammation is a driver could be a promising option for treatment and should be further tested. The next proposed perturbation was PRKACA (protein kinase cAMP-activated catalytic subunit alpha), with the same frequency as EP4. In the model, PRKACA exerts its effect mainly by regulating key survival pathways and regulators, such as the MAPKs and CREB1. While its involvement in the regulation of proliferative pathways and psoriasis has been documented (Gudjonsson et al, 2010), the prediction that it could serve as an additional target that could reduce keratinocyte hyperproliferation in psoriasis is novel and not yet described in the literature.

As $\mathrm{NF \kappa B}$ is a convergence point in the inflammatory response and directly regulates the transcription of many inflammatory genes, its direct inhibition or indirect inhibition through its regulators is no surprise as this would restore a more normal phenotype in KCs. The importance of NFאB as a key transcription factor in chronic inflammatory diseases, including psoriasis (Goldminz et al, 2013) has made NFKB and its regulators an attractive target for treatment in many diseases (Gilmore \& Herscovitch, 2006). Its inhibition, in combination with the inhibition of other transcription factors such as STAT3, has also been explored (Andrés et al, 2013). While the combination of NFאB and STAT3 is not detected as an impactful perturbation, the inhibition of STAT3 singly has the next highest frequency in the list. The model proposes the inhibition of STAT3 as well as the inhibition of other entities that control many inflammatory genes that sustain the inflammatory vicious cycle of psoriasis and have been previously studied as potential drug targets. These entities also include the abundantly-studied IL-17 (Amin et al, 2018). Indeed, several biologics used in psoriasis are targeting IL-17 (Ly et al, 2019). At the same time, while IFN $\gamma$ exerts a significant influence on the system, it has been reported that its inactivation alone is not enough to convert the psoriatic phenotypes back to normal, while its inhibition together with other entities such as IL-17 appears to be more effective (Meephansan et al, 2017). At the bottom of the list, some perturbations suggest the targeting of PI3K/AKT pathway components together with PLC $\gamma$, both pathways linked to proliferation and cell survival (Castilho et al, 2013; Haase et al, 1997).

\section{Discussion}

In this study, we aimed to develop an executable logical model for investigating the regulation of keratinocyte physiology by pro-psoriatic cytokines that could be used to investigate the therapeutic mode of action of $\mathrm{cPLA}_{2} \alpha$ inhibitors in psoriasis and predict likely druggable combinatorial partners for future investigations. Our model, supported by primary experimental observations, suggests that $\mathrm{cPLA}_{2} \alpha$-dependent $\mathrm{PGE}_{2} / \mathrm{EP} 4$ signaling is important for maintaining the psoriatic phenotype of KCs under cytokine stimulation, thus providing a therapeutic mode of action for the $\mathrm{CPLA}_{2} \alpha$ inhibitor AVX001 in psoriasis. Furthermore, we suggest AVX001 and the topical antipsoriatic drug calcipotriol have overlapping and distinct mechanisms of disease resolution and predict beneficial therapeutic effects when used in combination.

The pathology of psoriasis involves an interaction between immune cells and epidermal KCs that is critical for maintaining the chronic disease state, with keratinocyte activation leading to the release of 
chemokines and cytokines that promote the infiltration and amplification of immune cells (Lowes et al.). In our analysis we have focused on the key players that drive a vicious cycle of inflammation: the eicosanoids produced by KCs and act on $\mathrm{KC}$ themselves or immune cells, Th1-derived cytokines, which are established activators of phospholipases and cause eicosanoid release from KCs (Sjursen et al, 2000; Thommesen et al, 1998), and Th17-derived cytokines, which have largely unknown effects on eicosanoid signaling.

Our experimental findings demonstrated the importance of Th17 regulated $\mathrm{PGE}_{2}$ release via cPLA $\mathrm{a}$ in the induction of proinflammatory cytokine expression in $\mathrm{KCs}$, and suggest a role in cell survival. The dependence of IL-17 responses on $\mathrm{PGE}_{2}$ signaling was previously shown in normal human epidermal KCs and demonstrated to involve the activation of the MAPK pathway (Kanda et al, 2004, 2005). Since $\mathrm{cPLA}_{2} \mathrm{a}$ is a well-known target of the MAPK pathway (reviewed in (Clark et al, 1995)), this presents a putative mechanism for its regulation by Th17 cytokines. Th17 cytokines also upregulate PTGER4, which in turn can modulate cell survival and proliferation via PI3K (Peng et al, 2017) and ERK (Fujino et al, 2003) signaling. This suggests that Th17 cytokines may also regulate how the $\mathrm{KCs}$ respond to $\mathrm{PGE}_{2}$ and supports evidence that signaling via EP4 may predominate in psoriasis (Lee et al, 2019). Interestingly, calcipotriol suppressed the Th17-dependent release of $\mathrm{PGE}_{2}$, but not the up-regulation of PTGS2, and counteracted the changes in PTGER4 and PTGER2 expression. The suppressive effects of calcipotriol on $\mathrm{PGE}_{2}$ release differs from previous studies showing that calcipotriol can stimulate, or augment $\mathrm{PGE}_{2}$ release in both KCs and immune cells (Ashcroft et al, 2020; Ravid et al, 2016) and is rather consistent with the inhibition of Th17-dependent pro-inflammatory responses as described by (Lovato et al, 2016). It will be interesting to investigate the mechanism of suppression of Th17-dependent PGE $_{2}$ release by VDR activation, given it may be a novel mechanism accounting for some of the anti-psoriatic effects of the compound. The experimental data thus suggest both the $\mathrm{cPLA}_{2} \mathrm{a}-\mathrm{COX} 2-\mathrm{PGE}_{2}$ synthesis pathway and $\mathrm{PGE}_{2} / \mathrm{EP} 4$ signaling pathways are active in psoriatic $\mathrm{KCs}$, with implications for both paracrine and autocrine signaling. The impact of autocrine EP4 signaling was further investigated using the psoKC model. Simulations revealed that the states of key inflammatory and proliferation markers were active, corroborating their reported state in psoriasis only in simulations where EP4 was activated. We thus hypothesized that activation of the $\mathrm{PGE}_{2} / \mathrm{EP} 4$ axis is involved in an intrinsic amplification loop that could enable or sustain the effects of psoriatic cytokines and that suppression of the $\mathrm{PGE}_{2} / \mathrm{EP} 4$ axis by inhibition of $\mathrm{cPLA}_{2} \alpha$ presents a putative mode of action for AVX001 in psoriasis. We further explored this hypothesis using the in vitro model system by analyzing the expression of a subset of the phenotypic marker genes assigned as outputs, and by exploring the behavior of the KCs in different conditions using our computation model.

Phenotypic characterization of the in vitro model showed its inability to recapitulate the hyperproliferative state of KCs in response to Th17 stimulation, which is a prominent feature of psoriatic skin. Evidence for the usefulness of such epidermis-equivalent cultures to recapitulate the hyperproliferative state of KCs in response to cytokine stimulation is lacking, indicating a potential requirement of either the dermal compartment or alternative immunological signaling molecules to recreate this aspect of the disease (reviewed in Desmet et al, 2017). The computational model, on the other hand, accurately predicted the hyperproliferative state of the KCs in response to Th17 cytokines, allowing us to investigate the potential implication of inhibiting the $\mathrm{cPLA}_{2} \mathrm{a} / \mathrm{PGE}_{2} / \mathrm{EP} 4$ axis for a wider range of psoriatic phenotypes. Furthermore, the psoKC model was able to accurately reproduce the majority of our in vitro observations, in addition to those reported in the literature. We further explored its predictive use by expanding the simulations of the system's response to additional stimuli that were not tested in the lab, namely IFN $\gamma$ and $\mathrm{TNF} \alpha$. The ability to capture a more representative cytokine microenvironment for psoriatic $\mathrm{KCs}$, along 
with the integration of the $\mathrm{CPLA}_{2} \mathrm{a} / \mathrm{PGE}_{2} / \mathrm{EP} 4$ axis gave us access to an in silico experimentation system that allowed us to test a wide range of stimuli, observe the propagation of signals through the system and predict the effects of mutations.

Logical models have been previously used to elucidate drugs' mechanisms of action and effectiveness (Béal et al, 2021; Traynard et al, 2017). In silico treatment with the drugs tested in vitro revealed distinct mechanisms of action and interactions between the $\mathrm{CPLA}_{2} \mathrm{a}$ inhibition and Vitamin D analogs, where the two drugs impacted different phenotypic aspects of KCs. Calcipotriol is able to have both an antiproliferative (Kristl et al, 2008; Liang et al, 2017) and a pro-apoptotic effect (Huang et al, 2019; Tiberio et al, 2009) in psoriatic KCs. The model predicted that calcipotriol, alone or in combination with $\mathrm{cPLA}_{2} \mathrm{a}$ inhibition, acts either through rescuing the differentiation phenotype and/or via the induction of apoptosis. Alternatively, the inhibition of $\mathrm{cPLA}_{2} \mathrm{a}$ signaling directly impacts the proliferative phenotype of the simulated psoriatic KCs, as already described by (Ashcroft et al, 2020). Additionally, as initially hinted by the in vitro experiments, the combination of the two treatments uncovered a common regulation of several system components related to both $\mathrm{KC}$ physiological state and inflammatory immune response.

While an extensive literature search was employed in order to refine the model in a way that it correctly predicts the experimental observations, at the molecular level, some observed discrepancies between observations and predictions could be attributed to various reasons. First, the discretization method of the experimental results together with the intrinsic abstraction of discrete models could lead to some loss of information about the state of an entity. For instance, we observed experimentally that calcipotriol treatment reduced the expression of Th-17- stimulated ILIB expression but the effect was not significant and therefore did not meet the threshold set during the discretization process for inhibition. At the same time, the model predicts that IL- $1 \beta$ will be completely inhibited by calcipotriol. This discrepancy could be explained by the inability of the logical model to distinguish between partial and full inhibition, or that the threshold for discretization based on the experimental observations was too stringent. Additionally, as a gatekeeper of inflammation, IL-1 $\beta$ is under a strict regulation involving post-translational cleavage of proIL-1 $\beta$, the interleukin $1 \beta$ protein precursor, for complete activation (Liu et al, 2016). The control of calcipotriol over this mechanism of activation has been described in other systems, such as hematopoietic stem cells (Wang et al, 2020) and is thus included in the PKN, but not captured by measuring gene expression experimentally. Similar reasoning could explain the wrongly predicted state for IL-8 after $\mathrm{CPLA}_{2} \mathrm{a}$ inhibition; $\mathrm{PGE}_{2}$ has been reported to regulate IL-8 gene transcription through epigenetic mechanisms in human astrocytoma (Venza et al, 2012), and a similar regulation could occur also in KCs. While there was an extensive effort to incorporate the regulatory interactions relevant to eicosanoid signaling reported in the literature, half of the discrepancies involve the state of markers upon treatment with $\mathrm{cPLA}_{2} \mathrm{a}$ inhibitors. In addition to the aforementioned discretization limitations and post-translational regulation issues, wrong predictions may also result from the focus of the model on $\mathrm{PGE}_{2}$ signaling and not signaling mediated by other eicosanoids, or from gaps in the current knowledge on how $\mathrm{cPLA}_{2} \mathrm{a}$-derived lipid signaling mediators influence $\mathrm{KCs}$.

We further demonstrated how a logical model can be used to better understand a system and its internal regulatory mechanisms. As proposed by Thieffry et al., the presence of negative circuits (i.e. negative feedback loops) in a model is expected to generate cyclic attractors (Thieffry, 2007), where the state of the nodes is oscillating (i.e. continuously alternation between active and inactive states). Remarkably, the model contained a very limited number of functional circuits. This attribute became apparent during the integration of psoriasis-specific information in the model. A characteristic example is the negative feedback loop between STATs and SOCSs, where STAT1 and STAT3 activate their inhibitors SOCS1 and 
SOCS3. However, the activation of SOCS1 and SOCS3 appears inadequate to completely inhibit the expression of STAT-downstream targets in psoriasis (Madonna et al, 2010), making this negative circuit non-functional in the context of psoriasis, and it was, therefore, removed from the model. This observation on the model's behavior proposes the deregulation of mechanisms that would otherwise limit the spread of inflammatory response that might contribute to the development of psoriasis. Furthermore, stochastic simulations together with value propagation analyses confirmed the distinct role of Th1 and Th17 cells in the pathophysiology of psoriasis. All results indicated that Th1-derived cytokines have a key role in stimulating and further enhancing immune responses by regulating the majority of immune markers and chemotactic eicosanoids, as described in (Albanesi et al, 2018). The activation of cytokines and chemokines related to the recruitment, survival, and maintenance of Th17 and Th22 subpopulations by Th1 cells may indicate that Th1 activation precedes the activation of Th17 and Th22 during the development of psoriasis or that Th1 activation functions to amplify Th17 and neutrophil responses, as proposed in (Kryczek et al, 2008). The value propagation results also corroborated the dominance of an IFN $\gamma$ gene signature, where IFN $\gamma$ dominated the regulation of markers and TNF $\alpha$ had a more limited impact. This observation supports the claims that $\mathrm{TNF} \alpha$ is a potentiator and amplifier of IFN $\gamma$ effects (Albanesi et al, 2018). The synergistic effect of TNF $\alpha$ with IL-17 has also been described (Chiricozzi et al, 2011). These integrative responses could explain why the influence of TNF $\alpha$ on other nodes is overlapping on regulations from other inputs. However, it is worth noting that TNF $\alpha$ plays an important role in the development of the disease and its targeting remains a promising therapeutic option, but mainly due to its effect on Th17 cells (Furiati et al, 2019; Yost \& Gudjonsson, 2009).

Predictive logical models have been previously used to propose treatment strategies that could affect the system in a desired way. The suggested perturbations included both known and novel targets, some of them already involved in well-established psoriasis treatments. For the rest of the targets, inhibitory agents are available, and sometimes even approved as drugs, possibly opening attractive opportunities for drug repurposing. The fact that many of the suggested perturbations concern already used or explored targets confirms that the model sufficiently integrates the role of those entities in the system and is able to recognize the importance of certain stimuli and pathways to the progression of the disease. For example, VDR activation by the use of Vitamin D analogs and the blocking of IL-17 signaling are widely used in the treatment of psoriasis (Kim, 2010; Ly et al, 2019, 17). The importance of the $\mathrm{CPLA}_{2} \mathrm{a} / \mathrm{PGE}_{2} / \mathrm{EP} 4$ axis was further highlighted by the proposal of components of the pathway as a way to restore a normal phenotype. The role of EP4 was previously speculated to be involved in psoriasis and KCs but is not fully explored for its potentials for treating the disease. Therefore, we further propose the exploration of the role of EP4 in psoriasis and its potential as a drug target. Another entity, the catalytic subunit of Protein Kinase A (PRKACA), was proposed as a promising drug target. PRKACA's downstream effects involve multiple pathways, including WNT signaling, a pathway directly related with cPLA2a signaling (Xu et al, 2019), and psoriasis in general (Gudjonsson et al, 2010). PRKACA has also been studied in the context of cancer, where its aberrant regulation and overexpression have been related to inflammatory activation of Caspase 1, oncogene activation, and elevated PGE2 levels (Almeida et al, 2011). In addition to its role in hyperproliferation and inflammation, PRKACA has been associated with drug resistance in breast cancer by supporting the restoration of anti-apoptotic phenotypes of cancer cells (Moody et al, 2015). The current knowledge on the activity of the kinase, together with the model's predictions on the effects of its inhibition in psoriasis, suggest that its exact role in the development of the disease, together with its potential importance as a prognostic marker and/or drug target should be further explored.

While the model proposes certain perturbations, the need for further assessment of the results based on current knowledge and their actual experimental testing remains. For instance, the targeting of PLC $\gamma$ 
together with components of the PI3K/AKT pathway is proposed by the model. This comes as no surprise, as their action affects proliferation and cell survival. However, PLC $\gamma$ has been described as 'undruggable' in the literature (Lattanzio et al, 2013), as the development of small molecule inhibitors against it appears troublesome. The blocking of IFN $\gamma$ was also among the proposed perturbations. Even though its importance on the progression of the disease is supported by both the model and prior knowledge, targeting of IFN $\gamma$ on its own fails to restore a normal phenotype (Meephansan et al, 2017). Despite the model providing some insights on intracellular communication by integrating the effects of immune cell-derived cytokines as stimuli and KC-derived secreted factors that influence immune cells, it likely covers only a fraction of the complex intra- and inter-cellular signaling that underlies psoriasis. It is, therefore, expected that some observations at the tissue level cannot be captured to their totality by single-cell models. While understanding the role and behavior of individual cells in the disease, multicellular models are likely to provide a more accurate representation of biology as they would capture emergent behaviors that arise from intercellular interactions and communications.

In conclusion, this paper demonstrates how a combination of in vitro and in silico models, both without doubt flawed in their accuracy of representing the system to be analyzed, can complement each other to test and generate hypotheses for characterizing the regulatory mechanisms and effects of stimuli in a cellular system. Use of the in silico model allowed us to interpret in vitro observations in a more holistic manner providing additional mechanistic details of drug actions, and led us to propose novel candidates of drug targets that should be further explored. Through this collaboration, we characterized the regulation of certain lipid mediators in psoriatic $\mathrm{KC}$, which together with the prior knowledge of the involvement of $\mathrm{cPLA}_{2} \mathrm{a}$ signaling revealed yet another layer of involvement of KCs in the chronic inflammatory loop of psoriasis. Additionally, we presented a computational framework to interpret the effect of chemical perturbations and various stimuli in a cellular network by exploring the differences between the mechanisms of actions of $\mathrm{cPLA}_{2} \mathrm{a}$ inhibitors and calcipotriol. A perturbation analysis revealed promising entities whose targeting could restore a normal phenotype in KCs. For many of these targets, inhibitory agents have already been described, and sometimes these are even available as approved drugs, possibly opening attractive opportunities for drug repurposing. The combination of model-based analyses showcases how a systems biology approach can support a better understanding of a disease, further drive hypotheses on its causes, and suggest new targets for potential treatment.

\section{Author contributions}

ET and MK designed the in silico approach. FA and BJ designed the in vitro approach. ET performed the in silico model development and analysis. FA designed and performed the in vitro experiments. ET and FA designed the model validation experiments. ET and FA wrote the manuscript with input from all authors.

\section{Acknowledgments}

The authors would like to acknowledge Denis Thieffry and Laurence Calzone for their support on the building and analysis of the logical model. 


\section{Conflict of interest}

BJ is a shareholder of Coegin Pharma AS. FA is an employee of Coegin Pharma AS. The other authors declare that they have no competing interests. The funders had no role in the design of the study; in the collection, analyses, or interpretation of data; in the writing of the manuscript, or in the decision to publish the results.

\section{References}

Albanesi C, Madonna S, Gisondi P \& Girolomoni G (2018) The Interplay Between Keratinocytes and Immune Cells in the Pathogenesis of Psoriasis. Front Immunol 9

Allen D, Winters E, Kenna PF, Humphries P \& Farrar GJ (2008) Reference gene selection for real-time rtPCR in human epidermal keratinocytes. J Dermatol Sci 49: 217-225

Almeida MQ, Tsang KM, Cheadle C, Watkins T, Grivel J-C, Nesterova M, Goldbach-Mansky R \& Stratakis CA (2011) Protein kinase A regulates caspase-1 via Ets-1 in bone stromal cell-derived lesions: a link between cyclic AMP and pro-inflammatory pathways in osteoblast progenitors. Hum Mol Genet 20: 165-175

Amin M, Darji K, No DJ, Bhutani T \& Wu JJ (2018) Review of IL-17 inhibitors for psoriasis. J Dermatol Treat 29: $347-352$

Andrés RM, Montesinos MC, Navalón P, Payá M \& Terencio MC (2013) NF-кB and STAT3 Inhibition as a Therapeutic Strategy in Psoriasis: In Vitro and In Vivo Effects of BTH. J Invest Dermatol 133: 2362-2371

Ashcroft FJ, Mahammad N, Midtun Flatekvål H, J. Feuerherm A \& Johansen B (2020) cPLA2 $\alpha$ Enzyme Inhibition Attenuates Inflammation and Keratinocyte Proliferation. Biomolecules 10: 1402

Béal J, Pantolini L, Noël V, Barillot E \& Calzone L (2021) Personalized logical models to investigate cancer response to BRAF treatments in melanomas and colorectal cancers. PLOS Comput Biol 17: e1007900

Benhadou F, Mintoff D \& del Marmol V (2019) Psoriasis: Keratinocytes or Immune Cells - Which Is the Trigger? Dermatology 235: 91-100

Benham H, Norris P, Goodall J, Wechalekar MD, FitzGerald O, Szentpetery A, Smith M, Thomas R \& Gaston H (2013) Th17 and Th22 cells in psoriatic arthritis and psoriasis. Arthritis Res Ther 15: R136

Boehncke W-H \& Schön MP (2015) Psoriasis. The Lancet 386: 983-994

Boniface K, Bak-Jensen KS, Li Y, Blumenschein WM, McGeachy MJ, McClanahan TK, McKenzie BS, Kastelein RA, Cua DJ \& de Waal Malefyt R (2009) Prostaglandin E2 regulates Th17 cell differentiation and function through cyclic AMP and EP2/EP4 receptor signaling. J Exp Med 206: $535-548$

Boniface K, Bernard F-X, Garcia M, Gurney AL, Lecron J-C \& Morel F (2005) IL-22 Inhibits Epidermal Differentiation and Induces Proinflammatory Gene Expression and Migration of Human Keratinocytes. J Immunol 174: 3695-3702

Boukamp P, Petrussevska RT, Breitkreutz D, Hornung J, Markham A \& Fusenig NE (1988) Normal keratinization in a spontaneously immortalized aneuploid human keratinocyte cell line. $J$ Cell Biol 106: 761-771

Castilho RM, Squarize CH \& Gutkind JS (2013) Exploiting PI3K/mTOR Signaling to Accelerate Epithelial Wound Healing. Oral Dis 19: 551-558

Cather JC \& Crowley JJ (2014) Use of Biologic Agents in Combination with Other Therapies for the Treatment of Psoriasis. Am J Clin Dermatol 15: 467-478

Chiricozzi A, Guttman-Yassky E, Suárez-Fariñas M, Nograles KE, Tian S, Cardinale I, Chimenti S \& 
Krueger JG (2011) Integrative Responses to IL-17 and TNF- $\alpha$ in Human Keratinocytes Account for Key Inflammatory Pathogenic Circuits in Psoriasis. J Invest Dermatol 131: 677-687

Chizzolini C \& Brembilla NC (2009) Prostaglandin E2: igniting the fire. Immunol Cell Biol 87: 510-511

Clark JD, Schievella AR, Nalefski EA \& Lin L-L (1995) Cytosolic phospholipase A2. J Lipid Mediat Cell Signal 12: 83-117

Deng Y, Chang C \& Lu Q (2016) The Inflammatory Response in Psoriasis: a Comprehensive Review. Clin Rev Allergy Immunol 50: 377-389

Desmet E, Ramadhas A, Lambert J \& Van Gele M (2017) In vitro psoriasis models with focus on reconstructed skin models as promising tools in psoriasis research. Exp Biol Med Maywood NJ 242: $1158-1169$

Ekman A-K, Bivik Eding C, Rundquist I \& Enerbäck C (2019) IL-17 and IL-22 Promote Keratinocyte Stemness in the Germinative Compartment in Psoriasis. J Invest Dermatol 139: 1564-1573.e8

Farkas A, Kemény L, Széll M, Dobozy A \& Bata-Csörgo Z (2003) Ethanol and acetone stimulate the proliferation of $\mathrm{HaCaT}$ keratinocytes: the possible role of alcohol in exacerbating psoriasis. Arch Dermatol Res 295: 56-62

Feuerherm AJ, Jørgensen KM, Sommerfelt RM, Eidem LE, Lægreid A \& Johansen B (2013) Platelet-activating factor induces proliferation in differentiated keratinocytes. Mol Cell Biochem 384: 83-94

Flobak Å, Baudot A, Remy E, Thommesen L, Thieffry D, Kuiper M \& Lægreid A (2015) Discovery of Drug Synergies in Gastric Cancer Cells Predicted by Logical Modeling. PLOS Comput Biol 11: e1004426

Four Parameter Logistic Curve online data analysis tool, MyAssays Ltd., 25 th October 2012, http://www.myassays.com/four-parameter-logistic-curve.assay

Fujino H, Xu W \& Regan JW (2003) Prostaglandin E2 induced functional expression of early growth response factor-1 by EP4, but not EP2, prostanoid receptors via the phosphatidylinositol 3-kinase and extracellular signal-regulated kinases. J Biol Chem 278: 12151-12156

Furiati SC, Catarino JS, Silva MV, Silva RF, Estevam RB, Teodoro RB, Pereira SL, Ataide M, Rodrigues V \& Rodrigues DBR (2019) Th1, Th17, and Treg Responses are Differently Modulated by TNF- $\alpha$ Inhibitors and Methotrexate in Psoriasis Patients. Sci Rep 9: 7526

Georgescu S-R, Tampa M, Caruntu C, Sarbu M-I, Mitran C-I, Mitran M-I, Matei C, Constantin C \& Neagu M (2019) Advances in Understanding the Immunological Pathways in Psoriasis. Int J Mol Sci 20

Gilmore TD \& Herscovitch M (2006) Inhibitors of NF- $\kappa$ B signaling: 785 and counting. Oncogene 25: 6887-6899

Gisondi P, Del Giglio M \& Girolomoni G (2017) Treatment Approaches to Moderate to Severe Psoriasis. Int J Mol Sci 18

Goldminz AM, Au SC, Kim N, Gottlieb AB \& Lizzul PF (2013) NF-кB: an essential transcription factor in psoriasis. J Dermatol Sci 69: 89-94

Gudjonsson JE, Johnston A, Stoll SW, Riblett MB, Xing X, Kochkodan JJ, Ding J, Nair RP, Aphale A, Voorhees JJ, et al (2010) Evidence for altered Wnt signaling in psoriatic skin. J Invest Dermatol 130: $1849-1859$

Haase I, Liesegang C, Binting S, Henz BM \& Rosenbach T (1997) Phospholipase C-Mediated Signaling Is Altered During HaCaT Cell Proliferation and Differentiation. J Invest Dermatol 108: 748-752

Hangai S, Ao T, Kimura Y, Matsuki K, Kawamura T, Negishi H, Nishio J, Kodama T, Taniguchi T \& Yanai H (2016) PGE2 induced in and released by dying cells functions as an inhibitory DAMP. Proc Natl Acad Sci U S A 113: 3844-3849

Hellemans J, Mortier G, De Paepe A, Speleman F \& Vandesompele J (2007) qBase relative quantification framework and software for management and automated analysis of real-time quantitative PCR data. Genome Biol 8: R19

Hernandez C, Thomas-Chollier M, Naldi A \& Thieffry D (2020) Computational Verification of Large Logical Models-Application to the Prediction of T Cell Response to Checkpoint Inhibitors. Front Physiol 11 
Hinitt CAM, Benn TM, Threadgold S, Wood J, Williams AC \& Hague A (2011) BAG-1L promotes keratinocyte differentiation in organotypic culture models and changes in relative BAG-1 isoform abundance may lead to defective stratification. Exp Cell Res 317: 2159-2170

Hirabayashi T, Murayama T \& Shimizu T (2004) Regulatory mechanism and physiological role of cytosolic phospholipase A2. Biol Pharm Bull 27: 1168-1173

Huang T-H, Lin C-F, Alalaiwe A, Yang S-C \& Fang J-Y (2019) Apoptotic or Antiproliferative Activity of Natural Products against Keratinocytes for the Treatment of Psoriasis. Int J Mol Sci 20

Huwiler A, Feuerherm AJ, Sakem B, Pastukhov O, Filipenko I, Nguyen T \& Johansen B (2012) The $\omega 3$-polyunsaturated fatty acid derivatives AVX001 and AVX002 directly inhibit cytosolic phospholipase A(2) and suppress PGE(2) formation in mesangial cells. Br J Pharmacol 167: 1691-1701

Jan R \& Chaudhry G-S (2019) Understanding Apoptosis and Apoptotic Pathways Targeted Cancer Therapeutics. Adv Pharm Bull 9: 205-218

Kamata M \& Tada Y (2020) Efficacy and Safety of Biologics for Psoriasis and Psoriatic Arthritis and Their Impact on Comorbidities: A Literature Review. Int J Mol Sci 21

Kanda N, Koike S \& Watanabe S (2005) IL-17 suppresses TNF- $\alpha$-induced CCL27 production through induction of COX-2 in human keratinocytes. J Allergy Clin Immunol 116: 1144-1150

Kanda N, Mitsui H \& Watanabe S (2004) Prostaglandin E2 suppresses CCL27 production through EP2 and EP3 receptors in human keratinocytes. J Allergy Clin Immunol 114: 1403-1409

Khodapasand E, Jafarzadeh N, Farrokhi F, Kamalidehghan B \& Houshmand M (2015) Is Bax/Bcl-2 Ratio Considered as a Prognostic Marker with Age and Tumor Location in Colorectal Cancer? Iran Biomed J 19: 69-75

Kim GK (2010) The Rationale Behind Topical Vitamin D Analogs in the Treatment of Psoriasis. J Clin Aesthetic Dermatol 3: 46-53

Konger RL, Billings SD, Prall NC, Katona TM, DaSilva SC, Kennedy CRJ, Badve S, Perkins SM \& LaCelle PT (2009) The EP1 subtype of Prostaglandin E2 Receptor: Role in Keratinocyte Differentiation and Expression in Non-Melanoma Skin Cancer. Prostaglandins Leukot Essent Fatty Acids 81: 279-290

Konger RL, Brouxhon S, Partillo S, VanBuskirk J \& Pentland AP (2005) The EP3 receptor stimulates ceramide and diacylglycerol release and inhibits growth of primary keratinocytes. Exp Dermatol 14: 914-922

Konya V, Marsche G, Schuligoi R \& Heinemann A (2013) E-type prostanoid receptor 4 (EP4) in disease and therapy. Pharmacol Ther 138: 485-502

Kristl J, Slanc P, Krašna M, Berlec A, Jeras M \& Štrukelj B (2008) Calcipotriol Affects Keratinocyte Proliferation by Decreasing Expression of Early Growth Response-1 and Polo-like Kinase-2. Pharm Res 25: 521-529

Kryczek I, Wei S, Gong W, Shu X, Szeliga W, Vatan L, Chen L, Wang G \& Zou W (2008) Cutting Edge: IFN- $\gamma$ Enables APC to Promote Memory Th17 and Abate Th1 Cell Development. J Immunol 181: $5842-5846$

Lattanzio R, Piantelli M \& Falasca M (2013) Role of phospholipase C in cell invasion and metastasis. $A d v$ Biol Regul 53: 309-318

Lee J, Aoki T, Thumkeo D, Siriwach R, Yao C \& Narumiya S (2019) T cell-intrinsic prostaglandin E2-EP2/EP4 signaling is critical in pathogenic TH17 cell-driven inflammation. J Allergy Clin Immunol 143: 631-643

Lehmann B (1997) HaCaT Cell Line as a Model System for Vitamin D3 Metabolism in Human Skin. $J$ Invest Dermatol 108: 78-82

Leistad L, Feuerherm AJ, Faxvaag A \& Johansen B (2011) Multiple phospholipase A2 enzymes participate in the inflammatory process in osteoarthritic cartilage. Scand J Rheumatol 40: 308-316

Liang W, Lin Z, Zhang L, Qin X, Zhang Y \& Sun L (2017) Calcipotriol inhibits proliferation of human keratinocytes by downregulating STAT1 and STAT3 signaling. J Investig Med Off Publ Am Fed Clin Res 65: 376-381 
Linkous A \& Yazlovitskaya E (2010) Cytosolic phospholipase A2 as a mediator of disease pathogenesis: cPLA2 and disease pathogenesis. Cell Microbiol 12: 1369-1377

Liu J, Qian C \& Cao X (2016) Post-Translational Modification Control of Innate Immunity. Immunity 45: $15-30$

Lovato P, Norsgaard H, Tokura Y \& Røpke MA (2016) Calcipotriol and betamethasone dipropionate exert additive inhibitory effects on the cytokine expression of inflammatory dendritic cell-Th17 cell axis in psoriasis. J Dermatol Sci 81: 153-164

Lowes MA, Russell CB, Martin DA, Towne JE \& Krueger JG (2013) The IL-23/T17 pathogenic axis in psoriasis is amplified by keratinocyte responses. Trends Immunol 34: 174-181

Ly K, Smith MP, Thibodeaux Q, Reddy V, Liao W \& Bhutani T (2019) Anti IL-17 in psoriasis. Expert Rev Clin Immunol 15: 1185-1194

Madonna S, Scarponi C, Pallotta S, Cavani A \& Albanesi C (2012) Anti-apoptotic effects of suppressor of cytokine signaling 3 and 1 in psoriasis. Cell Death Dis 3: e334

Madonna S, Scarponi C, Sestito R, Pallotta S, Cavani A \& Albanesi C (2010) The IFN- $\gamma$-Dependent Suppressor of Cytokine Signaling 1 Promoter Activity Is Positively Regulated by IFN Regulatory Factor-1 and Sp1 but Repressed by Growth Factor Independence-1b and Krüppel-Like Factor-4, and It Is Dysregulated in Psoriatic Keratinocytes. J Immunol 185: 2467-2481

Magrioti V \& Kokotos G (2013) Phospholipase A2 inhibitors for the treatment of inflammatory diseases: a patent review (2010--present). Expert Opin Ther Pat 23: 333-344

Meephansan J, Subpayasarn U \& Ohtsuki MK and M (2017) Pathogenic Role of Cytokines and Effect of Their Inhibition in Psoriasis. Interdiscip Approach Psoriasis

Moody SE, Schinzel AC, Singh S, Izzo F, Strickland MR, Luo L, Thomas SR, Boehm JS, Kim SY, Wang ZC, et al (2015) PRKACA mediates resistance to HER2-targeted therapy in breast cancer cells and restores anti-apoptotic signaling. Oncogene 34: 2061-2071

Murakami M, Nakatani Y, Atsumi G, Inoue K \& Kudo I (2017) Regulatory Functions of Phospholipase A2. Crit Rev Immunol 37: 121-179

Murakami M, Yamamoto K \& Taketomi Y (2018) Phospholipase A2 in skin biology: new insights from gene-manipulated mice and lipidomics. Inflamm Regen 38: 31

Naini SM, Choukroun GJ, Ryan JR, Hentschel DM, Shah JV \& Bonventre JV (2016) Cytosolic phospholipase A2 $\alpha$ regulates G1 progression through modulating FOXO1 activity. FASEB J Off Publ Fed Am Soc Exp Biol 30: 1155-1170

Naini SM, Sheridan AM, Force T, Shah JV \& Bonventre JV (2015) Group IVA Cytosolic Phospholipase A2 Regulates the G2-to-M Transition by Modulating the Activity of Tumor Suppressor SIRT2. Mol Cell Biol 35: 3768-3784

Naldi A (2018) BioLQM: A Java Toolkit for the Manipulation and Conversion of Logical Qualitative Models of Biological Networks. Front Physiol 9: 1605

Naldi A, Hernandez C, Abou-Jaoudé W, Monteiro PT, Chaouiya C \& Thieffry D (2018) Logical Modeling and Analysis of Cellular Regulatory Networks With GINsim 3.0. Front Physiol 9

Napolitani G, Acosta-Rodriguez EV, Lanzavecchia A \& Sallusto F (2009) Prostaglandin E2 enhances Th17 responses via modulation of IL-17 and IFN- $\gamma$ production by memory CD4+ T cells. Eur $J$ Immunol 39: 1301-1312

Nicolaou A (2013) Eicosanoids in skin inflammation. Prostaglandins Leukot Essent Fatty Acids 88: $131-138$

Nograles KE, Zaba LC, Guttman-Yassky E, Fuentes-Duculan J, Suárez-Fariñas M, Cardinale I, Khatcherian A, Gonzalez J, Pierson KC, White TR, et al (2008) Th17 cytokines interleukin (IL)-17 and IL-22 modulate distinct inflammatory and keratinocyte-response pathways. $\mathrm{Br} J$ Dermatol 159: 1092-1102

Omland SH, Habicht A, Damsbo P, Wilms J, Johansen B \& Gniadecki R (2017) A randomized, double-blind, placebo-controlled, dose-escalation first-in-man study (phase 0) to assess the safety and efficacy of topical cytosolic phospholipase A2 inhibitor, AVX001, in patients with mild to moderate plaque psoriasis. J Eur Acad Dermatol Venereol JEADV 31: 1161-1167

Paulevé L (2017) Pint: A Static Analyzer for Transient Dynamics of Qualitative Networks with IPython 
Interface. In CMSB 2017 - 15th conference on Computational Methods for Systems Biology, Feret J \& Koeppl H (eds) pp 370-316. Darmstadt, Germany: Springer International Publishing

Peng X, Li J, Tan S, Xu M, Tao J, Jiang J, Liu H \& Wu B (2017) COX-1/PGE2/EP4 alleviates mucosal injury by upregulating $\beta$-arr1-mediated Akt signaling in colitis. Sci Rep 7: 1055

Perfetto L, Briganti L, Calderone A, Cerquone Perpetuini A, Iannuccelli M, Langone F, Licata L, Marinkovic M, Mattioni A, Pavlidou T, et al (2016) SIGNOR: a database of causal relationships between biological entities. Nucleic Acids Res 44: D548-554

Pfaff CM, Marquardt Y, Fietkau K, Baron JM \& Lüscher B (2017) The psoriasis-associated IL-17A induces and cooperates with IL-36 cytokines to control keratinocyte differentiation and function. Sci Rep 7: 15631

Pirkl M, Hand E, Kube D \& Spang R (2016) Analyzing synergistic and non-synergistic interactions in signalling pathways using Boolean Nested Effect Models. Bioinforma Oxf Engl 32: 893-900

Pozzi G, Guidi M, Laudicina F, Marazzi M, Falcone L, Betti R, Crosti C, Müller EE, DiMattia GE, Locatelli V, et al (2004) IGF-I stimulates proliferation of spontaneously immortalized human keratinocytes (HACAT) by autocrine/paracrine mechanisms. J Endocrinol Invest 27: 142-149

Rabeony H, Petit-Paris I, Garnier J, Barrault C, Pedretti N, Guilloteau K, Jegou J-F, Guillet G, Huguier V, Lecron J-C, et al (2014) Inhibition of Keratinocyte Differentiation by the Synergistic Effect of IL-17A, IL-22, IL-1 $\alpha$, TNF $\alpha$ and Oncostatin M. PLOS ONE 9: e101937

Ravid A, Shenker O, Buchner-Maman E, Rotem C \& Koren R (2016) Vitamin D Induces Cyclooxygenase 2 Dependent Prostaglandin E2 Synthesis in HaCaT Keratinocytes. J Cell Physiol 231: 837-843

Reinartz J, Bechtel MJ \& Kramer MD (1996) Tumor necrosis factor-alpha-induced apoptosis in a human keratinocyte cell line (HaCaT) is counteracted by transforming growth factor-alpha. Exp Cell Res 228: $334-340$

Ruijter JM, Lorenz P, Tuomi JM, Hecker M \& van den Hoff MJB (2014) Fluorescent-increase kinetics of different fluorescent reporters used for qPCR depend on monitoring chemistry, targeted sequence, type of DNA input and PCR efficiency. Microchim Acta 181: 1689-1696

Rundhaug JE, Simper MS, Surh I \& Fischer SM (2011) The role of the EP receptors for prostaglandin E2 in skin and skin cancer. Cancer Metastasis Rev 30: 465-480

Schoop VM, Mirancea N \& Fusenig NE (1999) Epidermal organization and differentiation of HaCaT keratinocytes in organotypic coculture with human dermal fibroblasts. J Invest Dermatol 112: 343-353

Se G, Rj A, A C, M D \& Pw G (2010) Evaluation of a range of anti-proliferative assays for the preclinical screening of anti-psoriatic drugs: a comparison of colorimetric and fluorimetric assays with the thymidine incorporation assay. Assay Drug Dev Technol 8: 389-400

Sjursen W, Brekke OL \& Johansen B (2000) Secretory and cytosolic phospholipase A(2)regulate the long-term cytokine-induced eicosanoid production in human keratinocytes. Cytokine 12: 1189-1194

Stoll G, Caron B, Viara E, Dugourd A, Zinovyev A, Naldi A, Kroemer G, Barillot E \& Calzone L (2017) MaBoSS 2.0: an environment for stochastic Boolean modeling. Bioinformatics 33: 2226-2228

Terskikh VV \& Vasil'ev AV (2005) Apoptosis and differentiation of epidermal keratinocytes. Russ J Dev Biol 36: 61-64

Thieffry D (2007) Dynamical roles of biological regulatory circuits1. Brief Bioinform 8: 220-225

Thommesen L, Sjursen W, Gåsvik K, Hanssen W, Brekke O-L, Skattebøl L, Holmeide AK, Espevik T, Johansen B \& Lægreid A (1998) Selective Inhibitors of Cytosolic or Secretory Phospholipase A2 Block TNF-Induced Activation of Transcription Factor Nuclear Factor- $\kappa \mathrm{B}$ and Expression of ICAM-1. J Immunol 161: 3421-3430

Tiberio R, Bozzo C, Pertusi G, Graziola F, Gattoni M, Griffanti P, Boggio P, Colombo E \& Leigheb G (2009) Calcipotriol induces apoptosis in psoriatic keratinocytes. Clin Exp Dermatol 34: e972-e974

Traynard P, Tobalina L, Eduati F, Calzone L \& Saez-Rodriguez J (2017) Logic Modeling in Quantitative Systems Pharmacology. CPT Pharmacomet Syst Pharmacol 6: 499-511 
Tsirvouli E, Touré V, Niederdorfer B, Vázquez M, Flobak Å \& Kuiper M (2020) A Middle-Out Modeling Strategy to Extend a Colon Cancer Logical Model Improves Drug Synergy Predictions in Epithelial-Derived Cancer Cell Lines. Front Mol Biosci 7

Tsuge K, Inazumi T, Shimamoto A \& Sugimoto Y Molecular mechanisms underlying prostaglandin E2-exacerbated inflammation and immune diseases. 10

Tuomi JM, Voorbraak F, Jones DL \& Ruijter JM (2010) Bias in the Cq value observed with hydrolysis probe based quantitative PCR can be corrected with the estimated PCR efficiency value. Methods San Diego Calif 50: 313-322

Venza I, Visalli M, Fortunato C, Ruggeri M, Ratone S, Caffo M, Caruso G, Alafaci C, Tomasello F, Teti $\mathrm{D}$, et al (2012) PGE2 induces interleukin-8 derepression in human astrocytoma through coordinated DNA demethylation and histone hyperacetylation. Epigenetics 7: 1315-1330

Viard-Leveugle I, Gaide O, Jankovic D, Feldmeyer L, Kerl K, Pickard C, Roques S, Friedmann PS, Contassot E \& French LE (2013) TNF- $\alpha$ and IFN- $\gamma$ Are Potential Inducers of Fas-Mediated Keratinocyte Apoptosis through Activation of Inducible Nitric Oxide Synthase in Toxic Epidermal Necrolysis. $J$ Invest Dermatol 133: 489-498

Wang X, Wang G, Qu J, Yuan Z, Pan R \& Li K (2020) Calcipotriol Inhibits NLRP3 Signal Through YAP1 Activation to Alleviate Cholestatic Liver Injury and Fibrosis. Front Pharmacol 11

Woo YR, Cho DH \& Park HJ (2017) Molecular Mechanisms and Management of a Cutaneous Inflammatory Disorder: Psoriasis. Int J Mol Sci 18

Xu H, Sun Y, Zeng L, Li Y, Hu S, He S, Chen H, Zou Q \& Luo B (2019) Inhibition of cytosolic phospholipase A2 alpha increases chemosensitivity in cervical carcinoma through suppressing $\beta$-catenin signaling. Cancer Biol Ther 20: 912-921

Yamamoto N, Koyama T, Nishino M, Iwasa S, Kondo S, Sudo K, Yonemori K, Yoshida T, Tamura K, Ozaki T, et al (2020) 534MO First in human study of ONO-4578, a PGE2-receptor EP4 antagonist, in monotherapy and combination with PD-1 checkpoint inhibitor nivolumab in patients with advanced or metastatic solid tumours. Ann Oncol 31: S468

Yao C, Sakata D, Esaki Y, Li Y, Matsuoka T, Kuroiwa K, Sugimoto Y \& Narumiya S (2009) Prostaglandin E2-EP4 signaling promotes immune inflammation through Th1 cell differentiation and Th17 cell expansion. Nat Med 15: 633-640

Yarla NS, Bishayee A, Vadlakonda L, Chintala R, Duddukuri GR, Reddanna P \& Dowluru KSVGK (2016) Phospholipase A2 Isoforms as Novel Targets for Prevention and Treatment of Inflammatory and Oncologic Diseases. Curr Drug Targets 17: 1940-1962

Yost J \& Gudjonsson JE (2009) The role of TNF inhibitors in psoriasis therapy: new implications for associated comorbidities. F1000 Med Rep 1

Ziv E, Rotem C, Miodovnik M, Ravid A \& Koren R (2008) Two modes of ERK activation by TNF in keratinocytes: Different cellular outcomes and bi-directional modulation by vitamin D. J Cell Biochem 104: 606-619 